\title{
AESMOTE: Adversarial Reinforcement Learning with SMOTE for Anomaly Detection
}

\author{
by \\ Xiangyu Ma \\ A thesis submitted to \\ the Faculty of Graduate Studies and Research \\ in partial fulfilment of \\ the requirements for the degree of \\ Master of Information Technology in Digital Media: specialization in \\ Data Science \\ Department of Information Technology \\ Carleton University \\ Ottawa, Ontario, Canada \\ April 2020
}

Copyright (c)

2020 - Xiangyu Ma 


\section{Abstract}

Intrusion Detection Systems (IDSs) play a vital role in securing today's Data-Centric Networks. In a dynamic environment that is vulnerable to various types of attacks, novel, fast, and robust solutions are in demand to handle fast changing threats and thus the ever-increasing difficulty of detection. In this dissertation, we present a novel reinforcement learning based anomaly detection algorithm that further enables anomaly-based intrusion detection. As anomaly detection frameworks are mostly supervised learning based, which seeks the advantage of stable predictions and good performance with pre-recorded datasets, we have further explored the performance of a combined framework of joining a reinforcement learning algorithm with classimbalance techniques. The motivation of this approach is to not only exploit the auto-learning ability from the reinforcement learning loop, but also correct the classimbalance problem, which is pervasive in existing solutions. Our proposed solution is developed based on AE-RL [1]. We further introduce an adapted SMOTE to address the class-imbalance problem while remodel the behaviors of the environmental agent for better performance. Experiments are conducted using NSL-KDD [2] datasets. Comparative evaluation and their results are presented and analyzed. Using techniques such as SMOTE, ROS, NearMiss1 and NearMiss2, performance measures obtained from our simulations have led us to recognize specific performance trends. The proposed model AESMOTE outperforms the original AE-RL in several cases. Experiment results show an Accuracy greater than 0.82 and F1 greater than 0.824. 


\section{Acknowledgments}

First and foremost, with my most sincere gratitude, I would like to acknowledge my supervisor Dr. Wei Shi for her dedication, patience, encouragement, and support. Without her guidance, knowledge and expertises, this dissertation would have not been possible.

I would also like to thank my family for their encouragement and support for my research interests and educational pursuits. 


\section{Table of Contents}

Abstract $\quad$ ii

Acknowledgments $\quad$ iii

Table of Contents $\quad$ iv

List of Tables $\quad$ vii

List of Figures viii

Nomenclature $\quad$ ix

1 Introduction 1

1.1 Background .......................... 1

1.1.1 Signature vs. Anomaly Detection . . . . . . . . . . . 2

1.1.2 Anomaly-based Intrusion Detection . . . . . . . . . . . . . . . 4

1.1.3 Malwares ....................... . . . 9

1.2 Reinforcement Learning . . . . . . . . . . . . . . . . 11

1.2.1 Model-free vs. Model-based . . . . . . . . . . . . . . . . . 14

1.2.2 Q-learning .......................... 15

1.2.3 Deep Q-learning. . . . . . . . . . . . . 16

1.2.4 Monte-Carlo Learning . . . . . . . . . . . . . . . 17

1.2.5 Temporal-Difference Learning . . . . . . . . . . . . . . . 18 
1.2.6 Actor-Critic Learning . . . . . . . . . . . . . . . . . 18

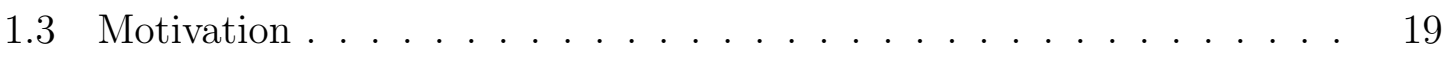

1.4 Problem Statement . . . . . . . . . . . . . . . . . . . 20

1.5 Major Contribution . . . . . . . . . . . . . . . 22

1.6 Thesis Overview . . . . . . . . . . . . . . . . . . . . . . . . 23

2 Related Work $\quad 24$

2.1 Related Work of Machine Learning Approaches on Anomaly Detection 24

2.2 Related Work on Class-Imbalance . . . . . . . . . . . . . . . . 27

3 Models and Assumption 30

3.1 Dataset .............................. 30

3.1.1 Attack Categories . . . . . . . . . . . . . . 31

3.2 Algorithm Description . . . . . . . . . . . . . . . . 34

3.2.1 General Description . . . . . . . . . . . . . . . 34

3.2.2 Over-sampling and Under-sampling methods . . . . . . . . . . 37

3.2.3 Main Algorithm . . . . . . . . . . . . . . . . . 41

3.2.4 Models and Error Reduction . . . . . . . . . . . . . . . . 43

3.2.5 Algorithm Overview . . . . . . . . . . . . . . . . . 46

4 Evaluation and Result Analysis 48

4.1 Dataset Description . . . . . . . . . . . . . . . 48

4.2 Evaluation Metrics . . . . . . . . . . . . . . . . . 52

4.3 Tools . . . . . . . . . . . . . . . . . . . . . . . . 53

4.4 Parameter Setup . . . . . . . . . . . . . . . . 55

4.5 SMOTE Performance . . . . . . . . . . . . . . . . . 59

4.6 Other Performance . . . . . . . . . . . . . . . . 63

5 Conclusion and Future Work $\quad 66$ 
List of References 


\section{List of Tables}

3.1 Attack Categories . . . . . . . . . . . . . . . . . 32

3.2 NSL-KDD Feature Variables . . . . . . . . . . . . . . . . 33

3.3 RL Components (Classifier Agent) . . . . . . . . . . . . . . . 34

3.4 RL Components (Environment Agent) . . . . . . . . . . . . 36

4.1 Training Parameters ................ 56

4.2 Across Algorithms . . . . . . . . . . . . . . . . . 63 


\section{List of Figures}

1.1 Reinforcement Learning Loop . . . . . . . . . . . . . . . . . . 12

1.2 Exploration vs. Exploitation Tradeoff . . . . . . . . . . . . . . 13

3.1 Overestimates of Q-learning . . . . . . . . . . . . . . 44

3.2 Algorithm Structure . . . . . . . . . . . . . . . . 47

4.1 Subclasses . . . . . . . . . . . . . . . . . . . . . . . 49

4.2 Distribution of Categories . . . . . . . . . . . . . . . 49

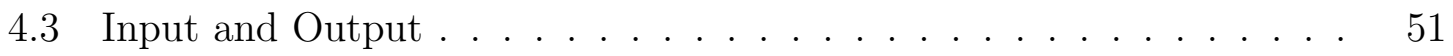

$4.4 \quad$ F1 scores for different number of episodes . . . . . . . . . . 56

4.5 Accuracy scores for different number of episodes . . . . . . . . 57

4.6 F1 score: 0.4033 for 10 episodes . . . . . . . . . . . . . . 57

4.7 F1 score: 0.642 for 50 episodes . . . . . . . . . . . . . . 58

4.8 F1 score: 0.824 for 100 episodes . . . . . . . . . . . . . . 58

4.9 F1-score Trending on SMOTE . . . . . . . . . . . . . . . 60

4.10 Confusion Matrix for AE-RL . . . . . . . . . . . . . . 61

4.11 Confusion Matrix for 70000 samples with SMOTE . . . . . . . . 61

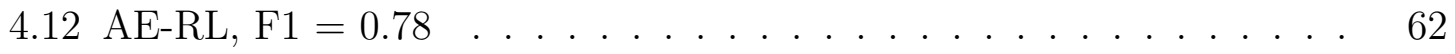

4.13 AESMOTE, $\mathrm{F} 1=0.8243 \ldots \ldots \ldots \ldots \ldots \ldots \ldots$

4.14 NearMiss1, $\mathrm{F} 1=0.7602 \ldots \ldots \ldots \ldots \ldots$

4.15 NearMiss2, $\mathrm{F} 1=0.7856 \ldots \ldots \ldots \ldots \ldots$

4.16 ROS, $\mathrm{F} 1=0.6932 \ldots \ldots \ldots \ldots \ldots \ldots \ldots \ldots \ldots$ 


\section{Nomenclature}

\begin{tabular}{|c|c|}
\hline Abbreviation & Explanation \\
\hline $\mathrm{AC}$ & Actor-Critic \\
\hline ADASYN & Adaptive Synthetic Sampling Approach for Imbalanced Learning \\
\hline $\mathrm{AE}$ & Auto-Encoder \\
\hline AE-RL & Adversarial Environment Reinforcement Learning \\
\hline AESMOTE & Adversarial Reinforcement Learning with SMOTE \\
\hline AIDS & Anomaly-based Intrusion Detection System \\
\hline AIPS & Anomaly-based Intrusion Prevention System \\
\hline $\mathrm{CA}$ & Classifier Agent \\
\hline $\mathrm{CNN}$ & Convolutional Neural Network \\
\hline DAD & Deep Anomaly Detection \\
\hline $\mathrm{DBN}$ & Deep Belief Network \\
\hline DNN & Deep Neural Network \\
\hline DDoS & Distributed Denial of Service \\
\hline
\end{tabular}




\begin{tabular}{|c|c|}
\hline DDQN & Double Deep Q-Network \\
\hline DoS & Denial of Service \\
\hline DP & Dynamic Programming \\
\hline DQN & Deep Q-Network \\
\hline DRL & Deep Reinforcement Learning \\
\hline EA & Environment Agent \\
\hline FQL & Fuzzy Q-Learning \\
\hline GAN & Generative Adversarial Network \\
\hline HMMs & Hidden Markov Models \\
\hline HRL & Hierarchical Reinforcement Learning \\
\hline IDS & Intrusion Detection System \\
\hline IoT & Internet of Things \\
\hline KNN & K-Nearest Neighbor \\
\hline LSTM & Long-Short Term Memory \\
\hline LR & Logistic Regression \\
\hline $\mathrm{MC}$ & Monte-Carlo \\
\hline MDP & Markov Decision Processes \\
\hline OPF & Optimal Power Flow \\
\hline $\mathrm{PCA}$ & Principle Component Analysis \\
\hline
\end{tabular}




\begin{tabular}{|c|c|}
\hline Probe & Probing Attack \\
\hline QoS & Quality of Service \\
\hline QoE & Quality of Experience \\
\hline $\mathrm{RL}$ & Reinforcement Learning \\
\hline $\mathrm{R} 2 \mathrm{~L}$ & Remote to User \\
\hline RNN & Recurrent Neural Network \\
\hline ROS & Random Over Sampling \\
\hline RUS & Random Under Sampling \\
\hline $\mathrm{RST}$ & Rough Set Theory \\
\hline SMOTE & Synthetic Minority Over-sampling TEchnique \\
\hline $\mathrm{STN}$ & Spacial Transformer Networks \\
\hline SVM & Support Vector Machine \\
\hline TD & Temporal Difference \\
\hline $\mathrm{U} 2 \mathrm{R}$ & User to Root \\
\hline
\end{tabular}




\section{Chapter 1}

\section{Introduction}

\section{$1.1 \quad$ Background}

Concerns about cyber security have emerged as a pressing issue not only in the scientific community but in society at large. It not only threatens the safety and security at the personal level but also at a much higher one [3]. For example, health care data breaches are growing threat to the health care industry causing not only data loss and monetary theft but also attacks on medical devices and infrastructure [4]. In a recent study, a whopping $94 \%$ of health care institutions reported having

been victims of cyberattacks [5]. In [6], it states that a deep understanding is required of technology's possibilities, and of mechanisms that can regulate and incentivize the development and use of technology that will maximize social welfare. On the economic side, an economic perspective is essential to understand the state of cybersecurity today. Several key economic challenges we are facing today are: misaligned incentives, information asymmetries, and externalities [7].

Computer communications first started in the early 1960s [8]. As it was large and expansive to acquire and run, companies, agencies, and researchers tend to rent time on the owner's machines. Security problems arose as the computer jobs were 
submitted by various users. Bernard Peters, Director of National Security Agency (NSA)'s "RYE" system, published a paper on "Security Considerations in MultiProgrammed Computer Systems", stated that security cannot be attained in an absolute sense in a multiprogramming system equipped with remote terminals and probability of loss should be considered especially for sensitive data [9]. As the number of technicians and communicators grew exponentially, people began to notice the severity of computer security problem. The US House of Representatives held three days of hearings on the threat that computers posed to privacy in 1966 [8]. The Defense Science Board demanded Willis H. Ware, the computer pioneer and RAND researcher, to lead a study group to examine the security problem. In the report published by the RAND Corporation in early 1970s, it stated that there is no secure open environment system with contemporary technology due to the exposure of consoles and communications [10]. As the viruses and hackings came in the 1980s, this problem was brought up to another level. Roger Schell, US Air Force's Lt. Col., pointed out in one of his articles that while security checkers tend to focus on the accidental flaws, the attackers placed the intrusion in ways that escapes detection. By the means of attackers, they can be any external interfaces [11]. The official responses to the security problem finally arrived in 1997, after being shaped by the outcomes

of a series of debates for the past three decades [8]. Hence, this may have caused a significant delay in the advances of cybersecurity researches.

\subsubsection{Signature vs. Anomaly Detection}

Common types of intrusion detection methodologies include signature-based detection, anomaly-based detection, and stateful protocol analysis [12].

Signature-based detection or misused detection systems focus on the idea of known threats. Existing attack descriptions or signatures have been recorded and 
compared with the incoming data stream to search for evidence of known threats. Examples of signatures are as follows: 1. A telnet attempt with a username of "root", which is a violation of an organization's security policy; 2. An e-mail with a subject of "Free pictures!" and an attachment filename of "freepics.exe", which are characteristics of a known form of malware; 3. An operating system log entry with a status code value of 645 , which indicates that the host's auditing has been disabled [12]. A common problem for Signature-based detection is that it lacks the ability of comprising multiple events as it cannot understand complex communications with limited signature database and only focuses on the current requested task. The main advantage of Signature-based detection is that it typically produces few false alarms, which provides high accuracy detection rates.

On the other hand, Anomaly-based detection systems focus on the process of comparing definitions of what activity is considered normal observed events to identify significant deviations [12]. Similar to the signature database, a profile is used to store all the normal behaviors of users, hosts, network connections, and applications. Observing the current activities, any significant deviations from the profile may be flagged as an anomaly. For example, an employee user at a company typically logs in around 9 a.m., at the company computer. His usual routines include reading emails, performing webpage transactions, updating clients' profiles and so on. If the system notices that this same user logs on the same computer at 2 a.m., transferring files, using compilers and debugging tools, which greatly differs from the profile, it will be flagged as a suspicious activity. The main advantage of anomaly-based detection is that it allows the system to detect previously unknown attacks. Its sensitivity to any new coming threats prevents, in the nick of time, malwares which potentially consume processing resources, send large number of emails, initiate large numbers of network connections, and perform other behavior that would significantly different from the 
established profiles for the computer [12]. However, this sensitivity advantage can also lead high false-alarm rate which causes unnecessary panics and over reactions.

\subsubsection{Anomaly-based Intrusion Detection}

The intrusion detection system (IDS) refers to identifying malicious activity in a computer-related system [13]. Anomalies usually refers to abnormalities, deviants, or outliers in the data mining and statistics literature [14]. In our daily lives, anomaly detection may appear in both research and application domains. Typical domains include fraud detection, medical anomaly detection, sensor networks anomaly detection, video surveillance, Internet of Things (IoT)big-data anomaly detection, industrial damage detection and cyber-intrusion detection [15]. We will briefly introduce the above-mentioned cases.

\section{Fraud Detection}

As defined by the Association of Certified Fraud Examiners (ACFE), fraud is the use of one's occupation for personal enrichment through the deliberate misuse or misapplication of the employing organization's resources or assets [16]. To be more simple, fraud is a deliberate act of deception to access valuable resources [17]. Typical applications of fraud detection include insurance, healthcare, banking, and telecommunication. Insurance fraud is the act of defrauding an insurance process, such as claiming a benefit without entitlement and denying a compensation on due. Common schemes include premium diversion, fee churning, asset diversion, and workers compensation fraud [18]. Traditional machine learning approaches had been applied for researches and produced decent performance [19] [18]. Healthcare fraud can also be referred as healthcare insurance fraud, occurs when a one defrauds an insurer health care plan. It plays a significant role in driving up costs in healthcare and thereby it is often taken as a serious problem. An example related to machine learning models 
on health care insurance fraud is presented in [20]. Banking fraud refers to the illegal ways of obtaining money, assets, and properties held by an owner or a financial institution. In banking fraud, credit card fraud is the most common scheme, including fraudulent activities such as theft of payment details and usage as a fraudulent source of funds. Some machine learning approaches related to banking fraud are shown in [21] [22]. Telecommunication fraud usually refers to the intention of illegally acquiring money and asset via telecommunication products. Typical machine learning approach include [23].

\section{Medical Anomaly Detection}

Due to the arise of big-data era, medical and Bioinformatics has becoming increasingly important. Significant amounts of biomedical data, including omics, image and signal data, have been accumulated, and the resulting potential for applications in biological and healthcare research has caught the attention of both industry and academia [24]. Anomaly detection tasks in medical field such as medical image analysis and clinical electroencephalography (EEG) records had great successes with the aid of deep learning techniques in preventing treatments for different medical conditions. These architectures produced outstanding performance using techniques such as Auto-Encoder(AE) [25], Convolutional Neural Network(CNN) [26], Deep Neural Network(DNN) [27], Deep Belief Network(DBN) [28], Long-Short Term Memory(LSTM) [29], Generative Adversarial Network(GAN) [30], and Recurrent Neural Network(RNN) [31].

\section{Sensor Networks Anomaly Detection}

Industrial wireless sensor networks(IWSN) have grew in recent year in both researches and industrial applications [32]. Due to the development of IWSN, security challenges had becoming a concern and are often dealt with intrusion detection system as a 
second line of defence against failure of normal network security protocols [33]. In [33], anomaly detection is separately defined to be parametric and non-parametric, where parametric methods require prior knowledge of the density distribution of the data being analysed and non-parametric methods can be applied without the knowledge of the density of distribution of data [34]. In parametric methods is usually performed with multivariate distribution functions [35] where as non-parametric methods usually can be performed by K-Nearest Neighbor(KNN) [36].

\section{Video Surveillance}

Video Surveillance, also known as Closed-Circuit Television(CCTV), refers to the act of monitoring designated areas of interest in order to ensure security [15]. Often times, applications in video surveillance produces large amount of unlabelled data. Detailed studies on the deep models of video anomaly dataset are being researched and categorized [37]. Another concern for video surveillance is that there isn't a unified definition of anomaly in real-life video surveillance when performing Deep Anomaly Detection(DAD). Methods had been used for further investigating DAD usage include Convolution Neural Network(CNN) [38], Recurrent Neural Network(RNN) [39], Spacial Transformer Networks(STN) [40], and Generative Adversarial Nets(GAN) [41].

\section{Internet of Things (IoT) Big-Data Anomaly Detection}

The Internet of Things(IoT) refers to a network of interconnected devices that forms a emerging communications paradigm in which devices have the ability to sense the surround environment and have ways to exchange data via Internet [42]. While the smart environment provides convenience to our daily lives, such as smart homes, smart healthcare, smart devices, smart services, and smart cities, it is also very susceptible to security attacks [43]. Some typical researches had been done on the IoT data, which mostly are generated by weather stations, Radio-frequency identification (RFID) tags, 
and IT infrastructure components [15]. These researches include CNN[44], LSTM [45], AE [46], DNN [47], and RNN [48].

\section{Industrial Damage Detection}

Systems such as turbines, power plants, high-temperature energy systems, storage devices and with rotating mechanical parts are the crucial industrial systems that greatly suffer from damages on a day-to-day basis [15]. Several machine learning techniques are concluded in [33]. Several deep learning models for detecting industrial damages are concluded in [49] [50].

\section{Anomaly Detection in Electric Power System}

Electric Power System is one of the critical infrastructure of our society and economy. As it evolves, many security problems could occur and IDS plays an important role in the procedure. In [51], a Principal Component Analysis (PCA) is proposed to determine the identities of input data for the optimal power flow (OPF), such as network configuration, generator capacity and system loads. Two subspaces are produced by the algorithm, which represents the regular and irregular space. An input data is detected to be an anomaly if the summed square of the data mapped onto the irregular subspace exceeds a statistical threshold. A recent work for IDS application in smart cities is presented in [52]. It proposed a new hybrid method D2H-IDS, which is an automated secure continuous cloud service availability framework for smart connected vehicles that enables an intrusion detection mechanism against security attacks and provides services that meet users' quality of service (QoS) and quality of experience (QoE) requirements. (Dataset: NSL-KDD + NS-3). In [53], 17 ensembled learning methods are covered and analysed on power system attack datasets, showed that ensemble learning is effective enhancement to basic learning methods and it is available approach to providing reliable detection results for cyber-attack in industrial 
control system (ICS) environment such as power system. In [54], Intrusion detection system using Online Sequence Extreme Learning Machine (OS-ELM) is proposed on the application on advanced metering infrastructure of smart grid. Large number of experiments are performed to determine the optimal algorithm parameters and the resulting OS-ELM-based intrusion detection system with other similar algorithms and the experimental results. [55] concludes a list of detection methodologies in Supervisory Control and Data Acquisition (SCADA) in a survey. [56] provided many case studies for the application of reinforcement learning in Cyber-Physical Systems such as Mobile Crowdsensing, Cognitive Radio Networks, and Mobile Edge Computing, as well as intrusion detection methods for smart grid.

\section{Anomaly Detection in Cyber Systems}

Since cybersecurity is the protection of computer systems from intrusions of hardware, software and data storage, different measures are required. The Anomaly-based Intrusion Detection System (AIDS) is a device or software that examines the actions that violate access control policy or packets that are suspicious by detecting average usage of the host resource or traffic of the network [57]. After detection, some systems may attempt to stop an intrusion. The Anomaly-based Intrusion Prevention System (AIPS) comes into play, which is considered an extension of IDS but also able to actively prevent or block intrusions that are detected [57]. In this dissertation, we focus on the detection of anomalies.

AIDS was first performed by sitting in front of a console and monitoring user activities. All the unusual activities are manually recorded down by the system administrators. Although it was somewhat effective in a limited user space, this early form of AIDS was not scalable and not efficient. Audit logs was the next step in late '70s and early '80s. System administrators print the audit logs to search for 
evidence of unusual or malicious behavior. Problem arises as it was still way too time consuming. Hence, audit logs were mainly used only to determine the cause of a particular security incident. Audit logs were later transferred to online storages for researchers to analyze data using developed programs. Nevertheless, the analysis was slow and often computationally intensive.

\subsubsection{Malwares}

Malware is defined as "a set of instructions that run on your computer and make your system do something that allow an attacker to make it do what he wants it to do" [58] . Put it to simple words, it consists of any software that is harmful to the system. Nowadays, malwares are often created by the hackers to seek secrets, to damage, disable computer systems, networks, mobile devices, tablets and so on. It also has becoming more of a nuisance due to the evolving weapon of choice for the attackers. Malwares may be launched via a number of anonymous methods such as insecure, open public wireless access point, which in return reduces the risk of identification, pursuit, and prosecution [59]. Multiple control systems could be controlled at once to help increase the efficiency for the attackers. For instance, Storm worm infection is a major intrusion, which ranged from one to ten million compromised systems [60]. Some general classifications of malware's form are as follows based on their functionality and replication method: Viruses: Self-replicating code that usually appear as an executable file (.exe). They tend to attach themselves to clean files and infect other files with their own bits of code. Most of the time, they are activated inadvertently by human interaction and the interaction with their creator disconnects once being released. Some typical examples include Melissa, Michelangelo, and Sobig [59].

Worms: Self-replicating code that infect the entire networks of devices, 
through network interfaces. They are usually activated without the need of human interaction. Known vulnerabilies are usually their breakthrough points. They are mostly used to destroy data and files and use infected machines to consecutively infect others. Updateable code and payloads are upcoming strategies, experimenting by their creators, which are exemplified by the Storm worm [60]. Some typical examples include Code Red, SQL Slammer, and Blaster [59].

Backdoors: A typically converted method that bypasses standard authentication to provide access to attackers. They are usually installed on compromised systems for future access and they rarely have self-replicating capability. Most of the time, they are a hidden part of a program [61]. Some typical examples include Back Orifice, Tini, and netcat [59].

Trojan Horse: A program that disguises itself as a legitimate while performing malicious behaviors in the background. The attacker behind a Trojan Horse is able to gain unauthorized access to steal data and monitor user actions. It also tends to create backdoors in the security control system to let other malwares invade. Some typical examples include tcpdump and Kazaa [59].

User-level Rootkit: A form of malware that stays hidden while providing administrator privileges to attackers. With user level of access, the rootkit can remove malicious processes from user-requested process lists. The core of operating system, however, is still not affected. Hence, tools and software can be used to detect rootkit. Some typical examples include Linux Rootkit (LRK) family and FakeGINA [59].

Kernel-level Rootkit: Different from user-level rootkit, kernel-level rootkit 
is able to affect the core of operating system and provide highest level of privileges to attackers. It still keeps its invisibility even from detection software. Additionally, investigators cannot remove kernel-level rootkits easily as it requires advanced technologies. Some typical examples include Adore and Hacker Defender [59].

Blended Malware: A combined version of features of malware which could have the disguise of a Trojan Horse, inserted through a backdoor applying an userlevel rootkit process and provide user level access to a remote control. Some typical examples include Lion and Bugbear [59].

\section{$1.2 \quad$ Reinforcement Learning}

Reinforcement learning (RL) [62] is a type of machine learning that possess the ability to self-learn and develop behaviors through trial-and-error simulations with a dynamic environment. Alongside supervised learning and unsupervised learning, reinforcement learning is the third paradigm of machine learning. The major key components of reinforcement learning consist of the agent, the action, the environment, and the reward state. The process starts at time $t$ with an agent taking an action, $a_{t}$, in an environment, which is being rewarded, $r_{t}$, and represented by a state, $s_{t}$. The state will be fed back to the agent for the recursive learning process.

The goal of the reinforcement learning is to find a path that maximize rewards. The general formula is set up to be:

$$
R_{t}=\sum_{k=0}^{\infty} \gamma_{k} r_{t+k}[63]
$$

where $R_{t}$ is the total accumulated, discounted, future return that the agent receives from time $t$ and on, $\gamma \in(0,1]$ the discount factor, and $r_{t+k}$ the rewards of each 


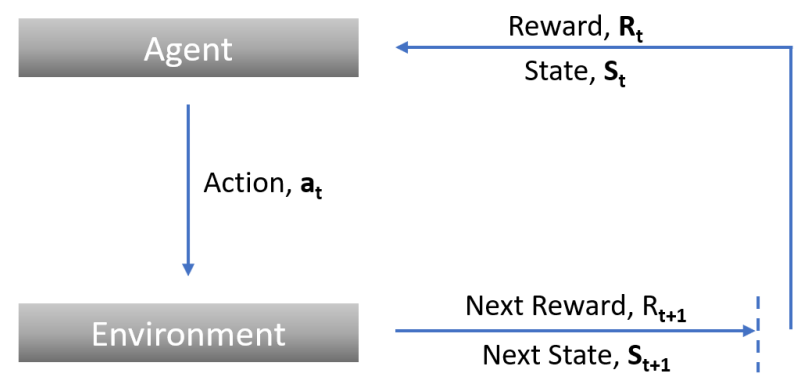

Figure 1.1: Reinforcement Learning Loop

future time step. This long-term calculation continues to go on for future time states repeatedly and discretely. Reinforcement learning introduces the value-function by taking its expectation at state $s, \mathrm{E}\left(R_{t} \mid s_{t}=s\right)$, denoted as $V^{\pi}(s)$, is showing how good is state $s$, for the agent to be in. The value function depends on the policy $\pi$ by which the agent chooses actions to behave. Among all possible functions, there exists an optimal value-function which has the highest value, denoted as $V^{*}(s)=\max _{\pi} V^{\pi}(s)$ and $\pi^{*}=\arg \max _{\pi} V^{\pi}(s)$ is the optimal policy which maximizes the action value achievable for state $s$. For convenient purpose, reinforcement learning set up a function called Q function, which takes inputs of state and action pair and outputs the value of rewards. Hence, we can rewrite the equation to be: $\pi^{*}=\arg \max _{a} Q^{*}(s, a)$, where $Q^{*}$ stands for the most optimal value for $Q$. According to Bellman equation, a recursive definition for optimal $\mathrm{Q}$ function is then defines to be:

$$
Q^{*}(s, a)=R(s, a)+\gamma E_{s^{\prime}}\left[V^{*}\left(s^{\prime}\right)\right]=R(s, a)+\gamma \sum_{s^{\prime} \in S} P\left(s^{\prime} \mid s, a\right) V^{*}\left(s^{\prime}\right)[63]
$$

where $R(s, a)$ is the immediate expected reward after performing action a at state $s$ and $\gamma E_{s^{\prime}}\left[V^{*}\left(s^{\prime}\right)\right]$ is the expected, discounted, accumulated, future reward after the transition to the next state $s^{\prime}$.

During the process of learning, there is a trade-off of between exploration and 


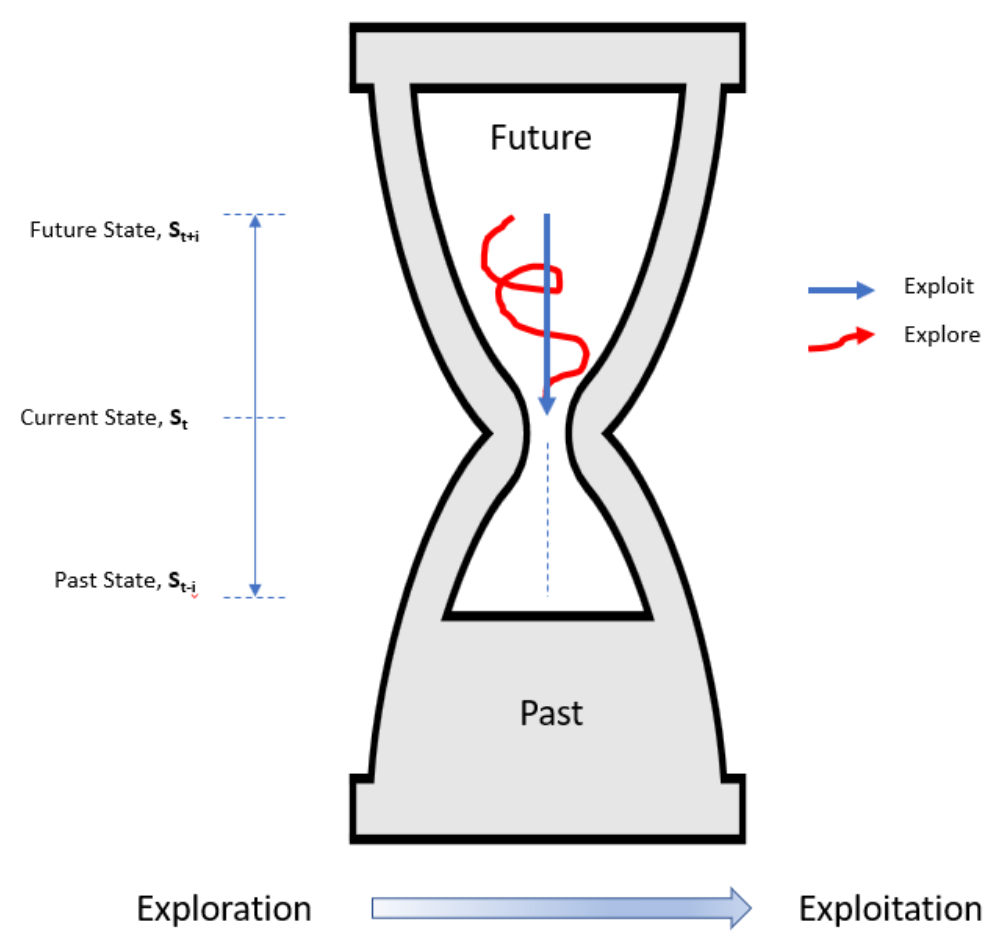

Figure 1.2: Exploration vs. Exploitation Tradeoff

exploitation. An agent needs to exploit from what it already knows to modify action policies in order to maximize rewards. On the other hand, it also needs to explore in order to make better action selections in the future. The dilemma is that neither exploration nor exploitation can be pursued exclusively without failing at the task. The agent must try a variety of actions and progressively favours those that appear to be best. On a stochastic task, each action must be tried many times to gain a reliable estimate its expected reward. The exploration-exploitation dilemma has been intensively studied by mathematicians for many decades [52]. The details of this concept are shown in Figure 1.2.

The rate of exploration and exploitation are presented by $\epsilon$ and $1-\epsilon$ in reinforcement learning, respectively, ranging from 0 to 1 . The exploration rate should start off in a high probability, often 1, and gradually decreases as the training goes on. When the training model is mature enough, that is reaching a decent prediction 
performance, the agent mostly makes decisions with its existing knowledge, based on the exploitation rate.

\subsubsection{Model-free vs. Model-based}

As mentioned previously, reinforcement learning requires a dynamic environment for agents to be interacted with. Depending on the types of environment, different methods can be chosen. Model-based method, is a method used when predictions can be made base on expected and prospective calculations according to the environment. On the other hand, Model-free method, is a method used when the system is constantly acquiring cached memories of circumstances and actions from retrospective experience. In reinforcement learning, control is another term for action. Instead of writing $\pi_{\theta}\left(a_{t} \mid s_{t}\right)$, where $\pi$ is the policy, beginning with action $a_{t}$, given state $s_{t}$, we can write it as $p\left(u_{t} \mid x_{t}\right)$, where $u, a$ and $s, x$ means the same. A trajectory of a sequence of states and controls can be expressed by $\tau=x_{1}, u_{1}, x_{2}, u_{2}, \ldots, x_{T}, u_{T}$. In Model-based method, model is the main component of the method, which learns the transition probability $T\left(s_{t+1} \mid s_{t}, a_{t}\right)$ from the current state $s_{t}$ and action $a_{t}$ to the next state $s_{t+1}$. Once the transition probability is learnt, it becomes helpful for agents to quickly identify and react when encounters similar situations in the future. Different from the model-free method, model-based method focuses on optimizing the trajectory with the least cost instead of maximizing rewards. However, model-based is impractical for a large state and action space since not all the transition probabilities can be learnt prospectively. On the other hand, model-free method obtains knowledge from the trial-and-error updates. Hence, it does not require to store all possible combinations of state and action pairs prospectively. We will discuss both methods in the following [64]. 


\subsubsection{Q-learning}

Q-learning was introduced by Watkins in 1989 [51]. It is a form of model-free reinforcement learning and can also be viewed as a method of asynchronous dynamic programming (DP). It provides agents with the capability of learning to act optimally in Markovian domains by experiencing the sequence of actions, without requiring them to build maps of the domains [51]. To always choose an action that maximize the discounted future reward, we would want to use some form of TD learning. A function $\mathrm{Q}$ is introduced to represent the maximum discounted future reward when an action is performed in a state and continually optimally from that point on. An intuitive way to think about this function is that it's the best possible score at the end of the game after performing an action in a state. The advantage of this method is that we can estimate the score at the end of the game knowing only the current state and action without any knowledge of future states and actions. The core of the algorithm is a value iteration update process, giving a learning rate for agent to learn the new coming $Q$ value.

$$
Q^{\text {new }}\left(s_{t}, a_{t}\right) \leftarrow(1-\alpha) Q\left(s_{t}, a_{t}\right)+\alpha\left(r_{t}+\gamma \max _{a} Q\left(s_{t+1}, a_{t+1}\right)\right)[63]
$$

The $\mathrm{Q}$ value is progressively being updated, until $s_{t+1}$ reaches the terminal state, in which the end of the episode. acts as a tuning factor, deciding the pace of learning speed, is always within the range of 0 and 1. A large $\alpha$ can lead to a faster learning process, yet may loses some information on the way. Similarly, verse versa. In the simplest case, the Q-learning is implemented as a table, with states as rows and actions as columns. Hence, to begin the process, the Q-table is initialized at random. Then, the agent starts to interact with the environment and upon each interaction, the agent will observe the reward of its action and the state transition. The agent computes the observed Q-value and updates its own estimates of Q. However, a problem would 
be that the agent is not exploring as it always reaches out for the closest best path without knowing the whole picture of the environment. Hence, random exploration is introduced. At some probability, the program will take a random action instead of taking the optimal action. This allows the agent to eventually figure out that there may be existence of some hidden rewards. We want the AI to eventually converge at some optimal policy, therefore we need to lower the probability of taking a random action over time as the agent becomes more confident with its estimates of Q-values.

\subsubsection{Deep Q-learning}

Reinforcement Learning optimizes an agent for sparse, time delayed labels called rewards in an environment. Markov Decision Processes (MDP) are a mathematical framework for modeling decisions using states, actions, and rewards. Q-learning is a strategy that finds the optimal action selection policy for any MDP. Although Q-learn ing is a powerful algorithm for policy selection, it does not have the ability to estimate values of unseen states. Hence, for an infinite state space, where environment setup may be changed, some Q-values cannot be calculated. We now introduce the Deep Q Network (DQN), which deals with this problem of lack of generality. A neural network is implemented, where the currents are the input and the estimates of $\mathrm{Q}$ values for each actions are the output. [65] The target Q-value for Q-learning is:

$$
r_{j}+\gamma_{a^{\prime}} Q\left(\phi_{j+1}, a^{\prime} ; \theta^{-}\right)[66]
$$

where $\phi$ is equivalent to state $s$ and $\theta$ is the parameters in the Neural Network. Since we are approximating Q-values, the goal is to minimize the error between target Q-value and the Q-value output from the network: 


$$
\text { Loss }=\left(y_{j}-Q\left(\phi_{j}, a_{j} ; \theta\right)\right)^{2}[66]
$$

A gradient descent is performed to find the minimal error. When training DQN, two essential techniques are involved:

Experience replay: This is a key technique behind many recent advances in deep reinforcement learning. Allowing the agent to learn from earlier memories can speed up learning and break undesirable temporal correlations [67]. It has been widely implemented in RL experiments and is shown to have a good performance for different algorithms such as actor-critic RL algorithms, DQN, double-Q learning algorithms.

Separate Target Network: When algorithm runs in loops using function approximation (i.e., Neural Network), bias may occur for a certain state action pair. In order to stabilize the trainings, the target network is reset to another for every few steps. This way, the Q-value would not face the problem of divergence.

\subsubsection{Monte-Carlo Learning}

In a model-free setup, we just focus on the value functions directly from the interactions with the environment. It involves predicting the value function of a certain policy without having a concrete model of the environment. Monte-Carlo Learning [68] learns the value functions directly from the episodes of experience. More specifically, we only acquire the reward information at the end of an episode $\tau=\left\{s_{1}, a_{1}, r_{1}, s_{2}, a_{2}, r_{2}, \ldots, s_{T}, a_{T}, r_{T}\right\}$. Monte-Carlo (MC) returns the means of the episode. There are two types of Monte-Carlo learning: first visit MC and every visit MC. The first visit MC takes the average of rewards only for the first time a state is visited in the episode. For instance, if state s occurred more than once in the trajectory sequence, we would jump over the repeated states, although their actions 
may be different. The every visit MC takes the average of rewards every time a state is visited in the episode. For the same case as above, we would take the average of reward till the end of the episode [68]. The reward function of Monte-Carlo learning is as follows:

$$
R_{t}=r_{t+1}+\gamma r_{t+2}+\gamma^{2} r_{t+3}+\ldots+\gamma^{T-t+1} r_{T}[68]
$$

\subsubsection{Temporal-Difference Learning}

Temporal-Difference Learning (TD) [69] refers to a model-free learning method, which updates the value of each state base on the prediction of the final return. More specifically, TD would take an action and update the value of the previous action base on the value of the current action. This method provides the advantage of updating values of more recent trends in order to more of the effect of a certain state. Comparing to $\mathrm{MC}$, TD has a lower variance as each update depends on less factors. However, MC has no bias as values are updated directly towards the final return while TD has some bias as values are updated towards each previous prediction. TD can be used for both the value function and the Q-function. The function for TD learning is as follows:

$$
R_{t}=r_{t+1}+\gamma V\left(s_{t+1}\right)[69]
$$

\subsubsection{Actor-Critic Learning}

When there are infinite input spaces and infinite output spaces, Actor-Critic Learning (AC) is introduced for this situation. The Actor-Critic Learning has two components: an actor and a critic. The actor takes in the current environment states and determines the best action to take from there. The critic plays the evaluation role by taking in the environment state and action, returning a score that represents how 
good is the action for the state. AC is advantageous to Policy Gradient method, as it tends to require much less training time than policy gradient methods. In the $\mathrm{AC}$ system, where the output of one network feeds into the input of the other, updating the parameters of the feeding network will shake up its output, which will propagate and be multiplied by any further changes through the end of the pipeline [70].

\subsection{Motivation}

The aspiration of this dissertation is to evaluate the performance and efficiency of a reinforcement learning model which consists of combination of class-imbalance techniques and environment control mechanism. The ultimate goal is to redirect anyone who wishes to apply the approach to discover the anomaly detection accuracy under different conditions in an organizational network.

Common approaches of anomaly detection frameworks are based on supervised and unsupervised learning methods. With the existing useful information of a labelled dataset, classification becomes the most used task in supervised learning. Through an efficient learning, such as neural network (ANN), support vector machine (SVM), Knearest neighbor (KNN), Naive Bayes, logistic regression (LR), Decision tree... etc., decent performance can occur and lead to high prediction accuracy. Nevertheless, the shortcoming of supervised learning is also obvious as real life datasets often come in unlabelled and labelling data manually is an expensive and time consuming task. On the other hand, unsupervised learning classifies data through unlabelled datasets, which are easy to obtain, yet the performance is usually inferior comparing to supervised learning classifiers. Hence, it is very common to see a trade-off between performance and accessibility for decision makers to take action. Most current supervised and unsupervised learning IDS are also limited to static data. As a fixed 
training process, they lack the adaptive learning skills for any changes to the dataset such as introduction of new labels and significant pattern changes to features. Another problem that arises for many types of datasets is due to class-imbalance. Data are said to suffer the class-imbalance problem when the class distributions are highly imbalanced [71]. In an imbalanced dataset, we might easily get an overall high accuracy, but minority classes suffer from a very low recall score. Typical examples include e-mail spam detection, medical diagnosis detection, and fraud detection. Imbalances may occur between classes, such as e-mail spam versus e-mail ham, diagnosis being positive versus negative, and fraud being normal versus abnormal. Imbalances may also occur within classes, such as e-mail spam containing a 9 to 1 ratio of advertisement versus scams, diagnosis of cancer containing 9 to 1 ratio of brain cancer versus liver cancer, and fraud detected containing a 9 to 1 ratio of trojan horse versus worms [72]. Additionally, false-positive error, in some cases, are not acceptable at all. For instance, if the cancer is regarded as positive and non-cancer as negative, failing to detect the diagnosis of cancer may cost a person's life. Hence, it is important to have a high accuracy for the minority class as well.

\subsection{Problem Statement}

To neutralize the above-mentioned problem of performance versus accessibility trade-off, Reinforcement Learning (RL) can be an efficient way to balance out their extremes. The fundamentals of RL establishes on: 1. Large and real-time datasets. RL can be applied to large and real-time datasets, which, in theory, could ultimately increase the performance of classifiers. 2. Agent is able to self-learn on its own: without any supervising activities during the learning process, which could self-label during the training phase based on its previous knowledge. Ideally, when data is large enough and in a real-time manner and without the consistent 
adjustment of labels, RL can be the better choice comparing to supervised and unsupervised learning. To solve the above-mentioned problem of imbalanced dataset, two conventional approaches are usually taken: the data-level approaches and algorithm-level approaches [73]. At the data-level, the original dataset is modified so that it becomes balanced enough to proceed further learning process. Re-sampling techniques are performed for this purpose and they are divided into over-sampling methods and under-sampling methods. At the algorithm-level, the objective is to make existing classifier adapt or strengthen on the learning of minority classes. In AE-RL [1], RL is integrated to generate new samples from a simulated environment and perform RL training on the simulated dataset. In addition to the conventional classifier agent, its features rely on the introduction of an environment agent, which is used to cope with the imbalance property. The trick is to set up an adversarial mechanism which provide a conflicting reward system of the two agents, so that the training dataset forms a fairly even distribution when being delivered to the classifier agent for training. This concept matches with the algorithm-level approach as mentioned above. In this dissertation, a data-level approach is implemented to further reduce the affect of class-imbalance. During the data simulation phase, the environment agent makes decision on choosing the type of data that should be delivered for training. The types are categorized into five categories: normal, Probing Attack (Probe), Denial of Service (DoS), Remote to User (R2L), and User to Root (U2R) [2]. For the minority classes, such as R2L and U2R, it is very likely that they are chosen multiple times for simulation, which forms the problem of duplicated data. Researchers in various disciplines have long recognized that aggregating study outcomes without assurance of sample independence violates a basic assumption of the meta-analytic method and may lead to erroneous conclusions [74]. We approach this problem by applying several over-sampling and under-sampling techniques to generate artificial data, assuring no existence of duplicated data. 
Hence, in this dissertation, the research questions are as summarized as follows:

RQ1. Conventional supervised and unsupervised learning are usually limited by manual labelling and prediction performance, respectively. Can we find an alternative solution to neutralize their extremes?

RQ2. Anomaly predictions are mostly limited by fixed training datasets. Can we test out the performance of our model on a dynamic dataset (i.e. data changes continuously)?

RQ3. Datasets are imbalanced, which causes high false-positive rates on minority classes. How do we reduce the imbalance biasses and increase prediction performance of those minority classes?

\subsection{Major Contribution}

As RL has the potential of possessing advantages over both supervised and unsupervised learning, in this dissertation, a RL framework is built and tested on its ability to detect anomalies in networks. We use NSL-KDD dataset, on which dynamic trainings are carried out and various sampling techniques are implemented in the process to improve performance on minority classes. Our contribution is summarized as follows:

C1. Establishes a RL framework on anomaly detection in networks using NSL-KDD dataset. 
C2. Through simulation trials, we obtain comparative analysis of different oversampling and under-sampling techniques at data-level and receive the best response based on performance.

C3. Training occurs based on a recursive over-sampled/under-sampled dataset joining a feature-selection mechanism, providing a dynamic environment for performance evaluation.

C4. Formulate trendings for SMOTE performance on the AE-RL framework and provide comparative evaluation against AE-RL and other sampling techniques, resulting in a better performance.

\subsection{Thesis Overview}

In Chapter 2, we introduce related works from all different aspects, including machine learning approaches on anomaly detection, class-imbalance techniques and relevance of this work to the industry. In Chapter 3, we set up assumptions, models, and algorithm descriptions. In Chapter 4, we present the simulation setup, the implementation of dataset and the result of analysis. Finally, in Chapter 5, we conclude this dissertation by recapitulating our whole research and some comparative evaluation will be shown. 


\section{Chapter 2}

\section{Related Work}

\subsection{Related Work of Machine Learning Ap- proaches on Anomaly Detection}

Anomaly Models, where each consists of assumptions, type of model architectures, computational complexity, and advantages and disadvantages [15]. Supervised anomaly detection techniques are superior in performance compared to unsupervised since these techniques use labeled samples [75]. Semi-supervised Deep Anomaly Detection (DAD) techniques assume that all training instances have only one class label. The assumption is that points which are close to each other both in input space and learned feature space are more likely to share the same label [15]. A few works of deep learning based semi-supervised techniques for anomaly detection is presented in [37] and [76]. Deep learning models learning models are widely used as feature extractors to learn robust features [77]. Hence, hybrid models are more scalable and computationally efficient due to the reduced input dimension. The downside of a hybrid model is that the learning within the hidden layer of feature extractor cannot be controlled since generic loss functions are employed instead of the customized objective for anomaly detection [15]. For unsupervised DAD, a 
few important assumptions are needed. First, the "normal" can be distinguished from "anomalous" regions in the latent feature space. Second, "normal" takes the majority of dataset. Lastly, outliers are identified based on intrinsic properties such as distances or densities. For example, in [78], the hidden layers of deep neural network aim to capture these intrinsic properties within the dataset. Another type of DAD is the Clustering based anomaly detection. The idea behind is to group data with similar patterns based on features extracted to detect new anomalies. [79] proposes a model to obtain the semantical presentations of normal and anomaly data to form clusters.

Anomaly detection with a dynamic environment often requires an adaptive learning strategy and RL possess such character. An early work of RL related research on intrusion detection is presented in [80]. The proposed algorithm tend achieve five objectives: 1. Online learning of attack patterns; 2. Rapid learning of data; 3. Extremely accurate in idenatifying priori attack patterns; 4 . Immediate identification of a priori attacks; 5. Ability to autonomously improve analysis. A Fuzzy Q-learning algorithm (FQL) is introduced in [81] to identify DDOS attacks. The training and testing are based on simulated datasets and the algorithm is compared against the Fuzzy Logic Controller algorithm [82] and Q-learning algorithm alone. [83] proposes an integration algorithm of Q-learning and Rough Set Theory (RST). The objective of this algorithm is to achieve maximum classification accuracy through a Q-learning process of obtaining the optimal cut value for RST to reduce attribute sets. A Monte-Carlo learning is displayed in [84] for the use of generating synthesized intrusion data based on a derived Poisson-Gamma joint probabilistic model. The proposed framework is to address the challenges of data scarcity and class-imbalance. [85] demonstrates a novel anomaly detection method based on TD learning, in which the reward function is based on the Markov decision process. It 
shows that TD learning can cope with complex sequential behaviors in datasets and it has the advantage of achieve good performance with limiting labeled data resource. [86] shows a kernel-based method using reinforcement learning to model behavior of a dynamic and sequential dataset. The predictions are evaluated based on reward signals. More specifically, the proposed method implements the Kernel Least-Squares Temporal-Difference (kernal LS-TD), showing that it has achieved better prediction accuracy against algorithms such as Hidden Markov Models (HMMs) and linear TD. Deep Reinforcement Learning (DRL) is fairly novel concept to the field, which had attracted significant interest due to its ability to learn complex behaviors in highdimensional data space. The advantage is that it does not need any assumptions and still achieves good performance. In [65], a design of time series anomaly detector using DRL is proposed. The model makes no assumption about the underlying mechanism of anomaly patterns, takes away threshold settings for simplicity and adapts well to dynamic environment. A recent work approaches the challenge of large-scale applications, which involves large state spaces and sparse delayed reward feedback [87] by using Hierarchical Reinforcement Learning (HRL). It's main strategy is to create subgoals in the system and identifies their corresponding rewards. Another novel framework uses Deep Actor-Critic Reinforcement Learning for sequential testing of anomaly detection [88]. The proposed method aims to dynamically detect anomalies based on posterior probabilities. [89] proposes a data poisoning attack method which takes a reverse perspective comparing to conventional IDS systems, showing that malicious workers, with limiting local information, still capable to find effective data poisoning attack strategies to interfere with crowd sensing systems. The proposed method is based on the deep reinforcement learning framework. [90] proposes a web attack detection system through the analysis of URLs andis deployed on edge devices. Various deep learning models are implemented and compared for evaluation. [91] proposes Vcash, a new reputation framework for tracking denial of 
traffic service in the Internet of connected vehicles. It borrows the idea of market trading and set up rules for the connected vehicles to follow. While restricting the malicious vehicle's spread of false message, it also performs traffic event monitoring and verification. Edge computing is mentioned in [92], introducing CloudSEC, a realtime lateral movement detection method, based on an evidence reasoning network for the edge-cloud environment. Descent performance shows that CloudSEC guarantees rapid and effective real-time attack detection. A data-driven model is proposed in [93] to achieve high-precision BGP based on deep learning methods. The model analyzes the routing behaviors without any prior knowledge.

\subsection{Related Work on Class-Imbalance}

Inappropriate evaluation metrics for model generated using imbalanced data can mislead to wrong conclusions. [94]. Common evaluation metrics include: Precision, Recall, F1 score, and Prediction accuracy. In this dissertation, since we are comparing our work to AE-RL, we choose to stay on F1 score as the standard measure. To solve an imbalanced dataset issue, countless sampling strategies had been developed throughout the years. The strategies aim to develop over-sampling and under-sampling techniques which smooth out the imbalanced property of the original dataset before any training is performed.

Random Over Sampling (ROS) [95] is the simplest techniques that works for all types of datasets. ROS extends the minority groups by duplicating them until the proportion of such minority groups reaches a predefined value. The advantage of this techniques is that it does not demand any prerequisite requirements on the original dataset. However, the technique is also simple enough to affect fraud detection performances. In [96], it stated that over-sampling with replication does not always 
improve minority class prediction. With replication of minority groups, over-fitting may occur as the decision region becomes too specific [97]. Hence, any new coming data that meant to belong to a minority group will be classified to others due to some trivial feature differences. On the other hand, Random Under Sampling (RUS) [97] randomly discards some majority group samples to balance the original dataset. The shortcoming appears for this technique when potential useful majority samples are dropped out during the process. Another under-sampling technique, NearMiss, is introduced early in [98] to improve prediction accuracy of minority classes. [99] later proposes a Boosted NearMiss Under-sampling algorithm on the training of SVMs (BNU-SVM), which aims to balance the dataset by adaptively updating weights over negative examples.

A different strategy to overcome the imbalance property is to generate artificial data samples, so called synthetic data generation. The Synthetic Minority Over-Sampling Technique (SMOTE) [100] proposes a technique that arbitrarily interpolates new minority samples in between several samples of that minority group, which can be found through K-nearest neighbors (KNN). This strategy avoided the over-fitting problem from ROS and still keeps the samples within the decision boundaries. A drawback for SMOTE is that the neighboring samples can be drawn from other minority groups, which may form overlapping regions among minority groups which increases additional noise. SMOTE is also not very practical in high dimensional datasets, as it does not attenuate the bias towards the classification in the majority class for most classifiers when data are high-dimensional, and it is less effective than RUS [101]. Only in cases when feature selection is applied, SMOTE can be beneficial for KNN classifiers in high dimensional datasets. In [102], it stated that samples near the borderlines of each groups are more apt to be misclassified than ones far from the borderlines, thus more important for classification. Hence, 
it proposes an extension of SMOTE, Borderline-SMOTE, which focus only on the oversampling of borderline samples. SMOTE-Boost, proposed in [103], combines the utilization of SMOTE for improving prediction of minority classes, and the standard boosting procedure, which maintains the accuracy for the majority classes. Another extension of SMOTE, Adaptive Synthetic Sampling Approach for Imbalanced Learning (ADASYN) [104], achieves two objectives: reducing bias of the imbalanced data distribution and adaptively learning difficult samples. 


\section{Chapter 3}

\section{Models and Assumption}

\subsection{Dataset}

In this dissertation, NSL-KDD is chosen for various reasons. First of all, it is wellorganized and cleansed. Second, we have observed that this dataset still contains imbalanced data as its previous version KDD-99. NSL-KDD [105] is the refined version of KDD-99, which eliminates redundant records of the original dataset for the purpose of reducing bias towards frequent records. Moreover, the dataset has a reasonable number of entries for researchers to affordably train the complete dataset rather taking a random small portion, which makes evaluation results consistent and thus easily comparable to other research works. Hence, although NSL-KDD may not be a perfect representation of the current real network, it is still a general admitted benchmark in the IDS research field. NSL-KDD consists of 41 features, including 38 continuous and 3 categorical variables, all being transformed, scaling down to the range of $[0-1]$ for the continuous ones and one-hot encoded to dummy variables for the categorical ones. After data transformation, the dataset consists of 122 features, including 38 continuous and 84 binary variables.

The dataset is split into a training set and testing set. The training set consists 
of 125973 samples and the test set consists of 22544 samples. The training set contains 23 different labels, which consists of the normal class and 22 different types of intrusion attacks. On the other hand, the testing set contains 38 different labels. The two sets have 21 labels in common, with 2 distinct labels in training set and 17 distinct labels in testing set. The dataset is further grouped into five major categories: NORMAL, PROBE, R2L, U2R and DoS [105]. In this dissertation, the challenge raised due to the distinct labels in the testing set will not be specifically dealt with as the final purpose is to predict the major categories for a given input.

\subsubsection{Attack Categories}

\section{DOS}

Denial of Service, or DOS, refers to the act of fulfilling the memory spaces and overloading the computing resources of working machines by sending unimportant information for the purpose of affecting the usage of legitimate users [105].

\section{PROBE}

Probing attacks, or PROBE, refers to the act of gather information of networks for the purpose of circumventing security controls. It may be not be directly defined as an attack, but rather it is a sign of future attacks [105].

\section{R2L}

Remote to Local, or R2L, refers to the act of accessing machines via sending set of packets over the network without any permission for the purpose of exploiting vulnerability to gain local access [105]. 
$\mathrm{U} 2 \mathrm{R}$

User to Root, or U2R, refers to the act of obtaining full access of machines by first accessing the network resources as a normal user [105].

The details of the label categorization is as follows:

Table 3.1: Attack Categories

DoS back, land, neptune, pod, smurf, teardrop, mailbomb, apache2, processtable, udpstorm

\begin{tabular}{cl}
\hline PROBE & ipsweep, nmap, portsweep, satan, mscan, saint \\
\hline R2L & ftp_write, guess_passwd, imap, multihop, phf, spy, \\
& warezclient, warezmaster, sendmail, named, \\
& snmpgetattack, snmpguess, xlock, xsnoop, worm \\
\hline U2R & buffer_overflow, loadmodule, perl, rootkit, httptunnel, \\
& ps, sqlattack, xterm \\
\hline
\end{tabular}

The initial dataset comes with three types of variables: binary, nominal, and continuous. The details of feature types as shown in the following: 
Table 3.2: NSL-KDD Feature Variables

\begin{tabular}{cl}
\hline Binary & Land, logged_in, root_shell, su_attempted, \\
& is_host_login, is_guest_login \\
\hline Nominal & Protocol_type, Service, Flag \\
& Duration, src_bytes, dst_bytes, wrong_fragment, urgent, \\
& hot, num_failed_logins, num_compromised, num_root, \\
& num_file_creations, num_shells, num_access_files, \\
& num_outbound_cmds, count, srv_count, serror_rate, \\
& srv_serror_rate, rerror_rate, srv_rerror_rate, same_srv_rate, \\
& diff_srv_rate, srv_diff_host_rate, dst_host_count, \\
& dst_host_srv_count, dst_host_same_srv_rate, \\
& dst_host_diff_srv_rate, dst_host_same_src_port_rate, \\
& dst_host_srv_diff_host_rate, dst_host_serror_rate, \\
& dst_host_srv_serror_rate, dst_host_rerror_rate, \\
& dst_host_srv_rerror_rate
\end{tabular}




\subsection{Algorithm Description}

\subsubsection{General Description}

As mentioned previously, RL has two advantages over conventional supervised learning: easy deal with large and real-time datasets and has the ability to learn on its own without any supervision. This section will introduce the general process of RL and the detailed structure of AE-RL along with techniques that associate with the class-imbalance concern. Instead of giving rules, correcting input/output pairs and finding similarities and differences between data points, reinforcement learning emphasizes on the performance, which bases on rewards and punishments as instructions for positive and negative behavior. The reward system is assigned to a software agent, who constantly interacts with the environment and learns from the acquired rewards and punishments. Supervised and unsupervised learning are usually one-shot, myopic, considering instant reward; while reinforcement learning is sequential, far-sighted, considering long-term accumulative reward [106].

In a RL framework, the agent, the state, the action, the environment, and the reward are components of the learning loop.

Table 3.3: RL Components (Classifier Agent)

\begin{tabular}{cl}
\hline Components & Corresponding Implementation \\
\hline Agent & Classifier \\
\hline Environment & Training dataset \\
\hline State & Current line of data \\
\hline Action & Making prediction of attack type \\
\hline Reward & Correctness of prediction \\
\hline
\end{tabular}


Table 3.3 defines the corresponding matches of RL components to our real dataset. During the active RL process, for every incoming data, it is noted as the current state. Making a prediction by the classifier agent would be regarded as taking an action. Receiving a feedback of whether the prediction is correct is defined as the reward. Correct predictions receive a value of +1 and incorrect predictions receive a value of -1 .

In this dissertation, Q-learning is chosen for the base learning algorithm. As mentioned previously in Chapter 1, Q-learning finds the maximum discounted accumulated reward for each state-action pair. In other word, for each incoming line of data, Q function will find all rewards for every possible action and accumulate the best ones in record. The Q-learning functions as follows:

$$
Q^{\text {new }}\left(s_{t}, a_{t}\right) \leftarrow(1-\alpha) Q\left(s_{t}, a_{t}\right)+\alpha\left(r_{t}+\gamma \max _{a} Q\left(s_{t+1}, a_{t+1}\right)\right)[63]
$$

In this equation, $s_{t}$ represents the current state, $a_{t}$ represents the current action, $s_{t}+1$ represents the next state, $a_{t}+1$ represents the next action, $\gamma$ represents the discount factor, $\alpha$ represents the learning rate, $r_{t}$ represents the current reward, and $Q^{\text {new }}$ represents the updated $\mathrm{Q}$ value. In this dissertation, values of 1.0 and 0.001 for $\alpha$ and $\gamma$ are assumed, respectively, based on the assumption that all individual data are independent.

Now, the question comes to which data we should give for the agent to train. If, let's say, the original training set is 100 times larger, running through the complete dataset would be non-realistic. Even if we have enough computation power to train the complete dataset, the imbalance problem is still existence. Hence, a mechanism is constructed to handle this problem, noted as the environment agent. Similar to the classifier agent, the environment agent also performs Q-learning for every incoming 
data. The difference is that it accumulates opposite rewards as the classifier agent. For instance, when the incoming NORMAL data is predicted to be normal by the classifier agent, the environment agent receives a negative reward. The essential idea behind the environment agent is to learn the prediction performance of classifier agent and select the most appropriate categories of data for classifier agent to train next. Hence, the classifier agent is always forced to train the most difficult samples at the moment, which balances out the distribution of training samples. With the cooperation of environment agent, a more balanced dataset is simulated, accuracies may improve, especially for the minority classes such as U2R and R2L.

Table 3.4: RL Components (Environment Agent)

\begin{tabular}{cl}
\hline Components & Corresponding Implementation \\
\hline Agent & Environment \\
\hline Environment & Training dataset \\
\hline State & Current type of data \\
\hline Action & Making prediction of the type of next line of data \\
\hline Reward & Correctness of classifier prediction (opposite reward) \\
\hline
\end{tabular}

As described in table 3.4, the process begins with the current state being the current type of data, the current action being making prediction of the type of next line of data for training based on its policy, and receiving opposite amount of reward as classifier agent in memory.

One important thing to note is that there are two different epsilon value for the two agents as these values decide the learning exposure of agents to the incoming data. 


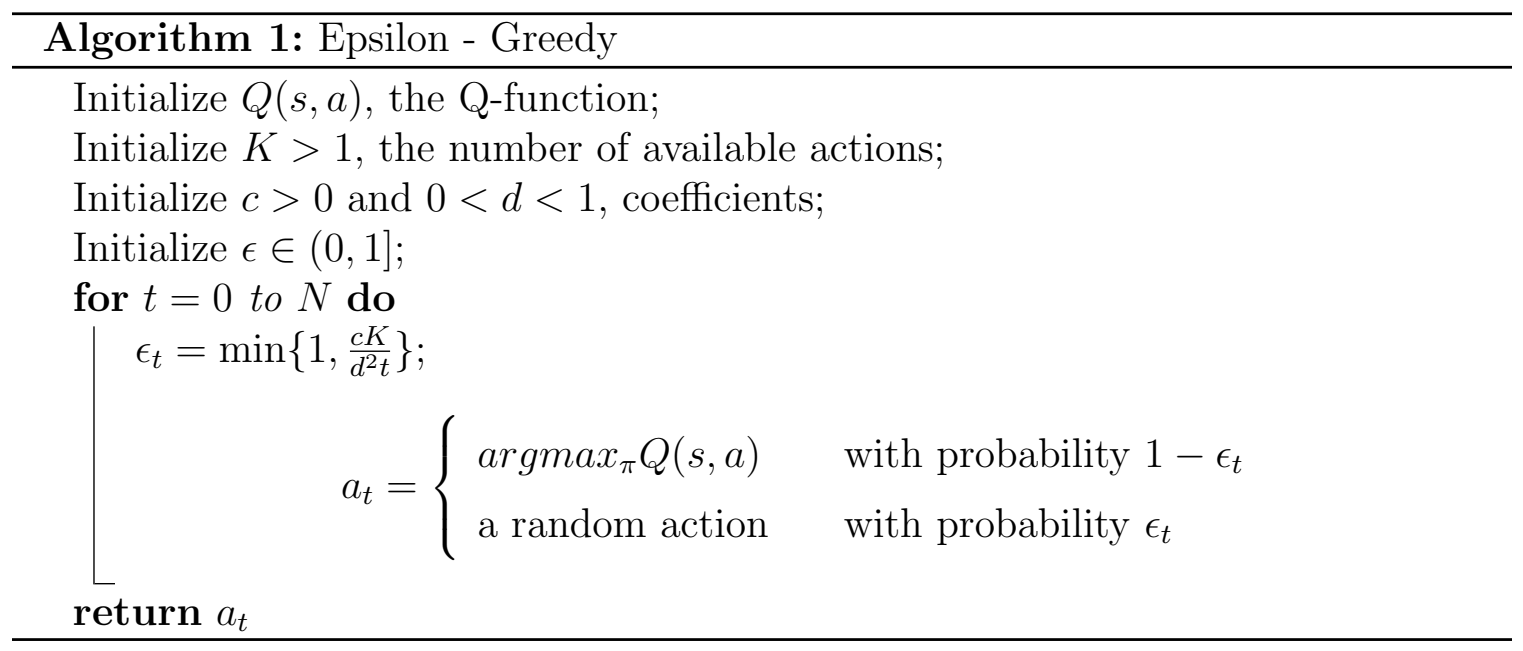

As we can see from Algorithm 1, $\epsilon$ first start off being equal to 1 and is dynamically changing in a descending trend as $t$ increases. Actions are then calculated by taking the piecewise choice based probability $\epsilon$. Two agents both have their epsilon estimates and are different for each case. The lower bound of epsilon for the classifier agent is relatively low as the model is becoming more and more stable, which is set to 0.01. We set this low value because classifier agent is our main agent as it's "the final decision maker" and we want it to have a high exploration rate. On the other hand, the lower bound of the epsilon for the environment agent is set as a hyperparameter [1], with an ideal value of 0.8. The reason for this value to be large is that there is no need for the environmental agent to be abolute ly accurate since it's based on an unstable factor. Additionally, all these values are selected from trial-and-error process.

\subsubsection{Over-sampling and Under-sampling methods}

Now, the question comes to the existence of duplicate data when selecting samples from the original dataset $D$. This problem may appear trivial for the majority classes such as NORMAL, DOS, and PROBE, but when dataset is small enough, like R2L and U2R, which only has around 1000 and 50 lines of data, respectively, it is 
very likely that data is being selected more than once. Hence, Over/Under-sampling techniques SMOTE, ROS, NearMiss1, and NearMiss2 are applied and compared. These techniques are chosen because of their simplicities of implementation and the fact that they take completely different approaches, which results in easier comparisons.

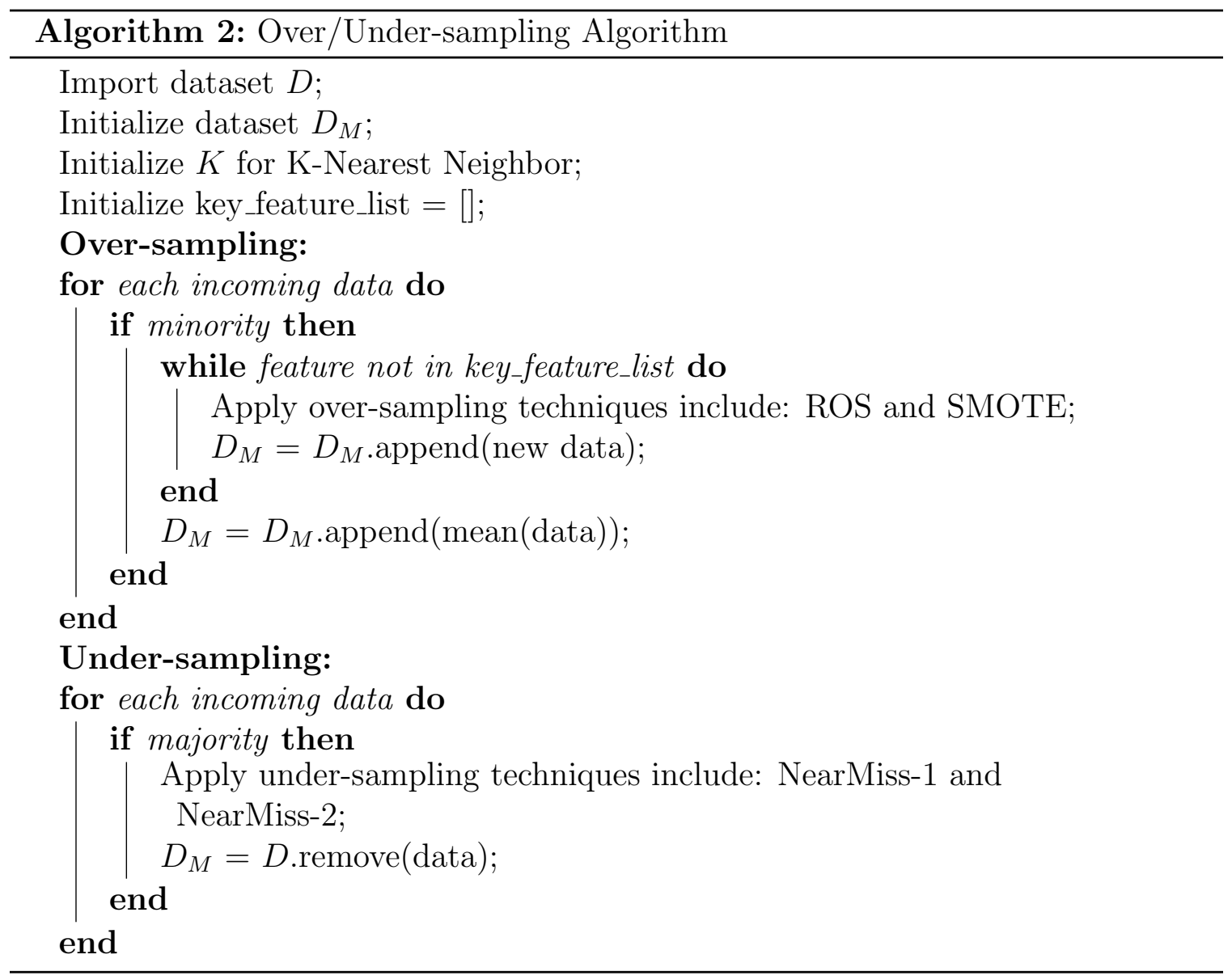

To begin, we need to initialize the value $K$ as the K-Nearest Neighbor, which will be used in all the following techniques. For Over-sampling, first we set R2L and U2R as our minority classes. According to [2], some key features such as "number of file creations" and "number of shell prompts invoked" can be carefully looked 
when over-sampling U2R data and "duration of connection", "service requested", and "number of failed login attempts" can play an significant role for R2L data. The essential idea is to keep the same values for these features when generating new dataset, while other unimportant features are randomly generated. The next step is applying different Over-sampling techniques.

\section{Random Over Sampling (ROS)}

Random Over Sampling (ROS) is the most naive approach to generate new samples. The new samples are randomly duplicated in the minority classes with replacement. They are referred "naive resampling" due to the fact that they assume nothing about the nature of data and no heuristics are used. The advantage, however, is that it is simple to implement and fast to execute.

\section{Synthetic Minority Over-Sampling Technique (SMOTE)}

Synthetic Minority Over-Sampling Technique (SMOTE) proposes the idea of generating synthetic samples based on existing ones. Based on the "feature space" of data, the minority class is over-sampled by generating new samples on the line segments formed by the endings of its $K$ nearest neighbours [100]. $K$ is chosen depending on the amount of over-sampling required. On each line segment, the new generated samples can determined by multiplying the difference between the feature vector and its nearest neighbour by a random number ranged from 0 to 1 . This approach allows generating synthetic samples that follows certain feature patterns and forces the boundaries of minority classes to become more general. A simple illustration of SMOTE is as follows: 


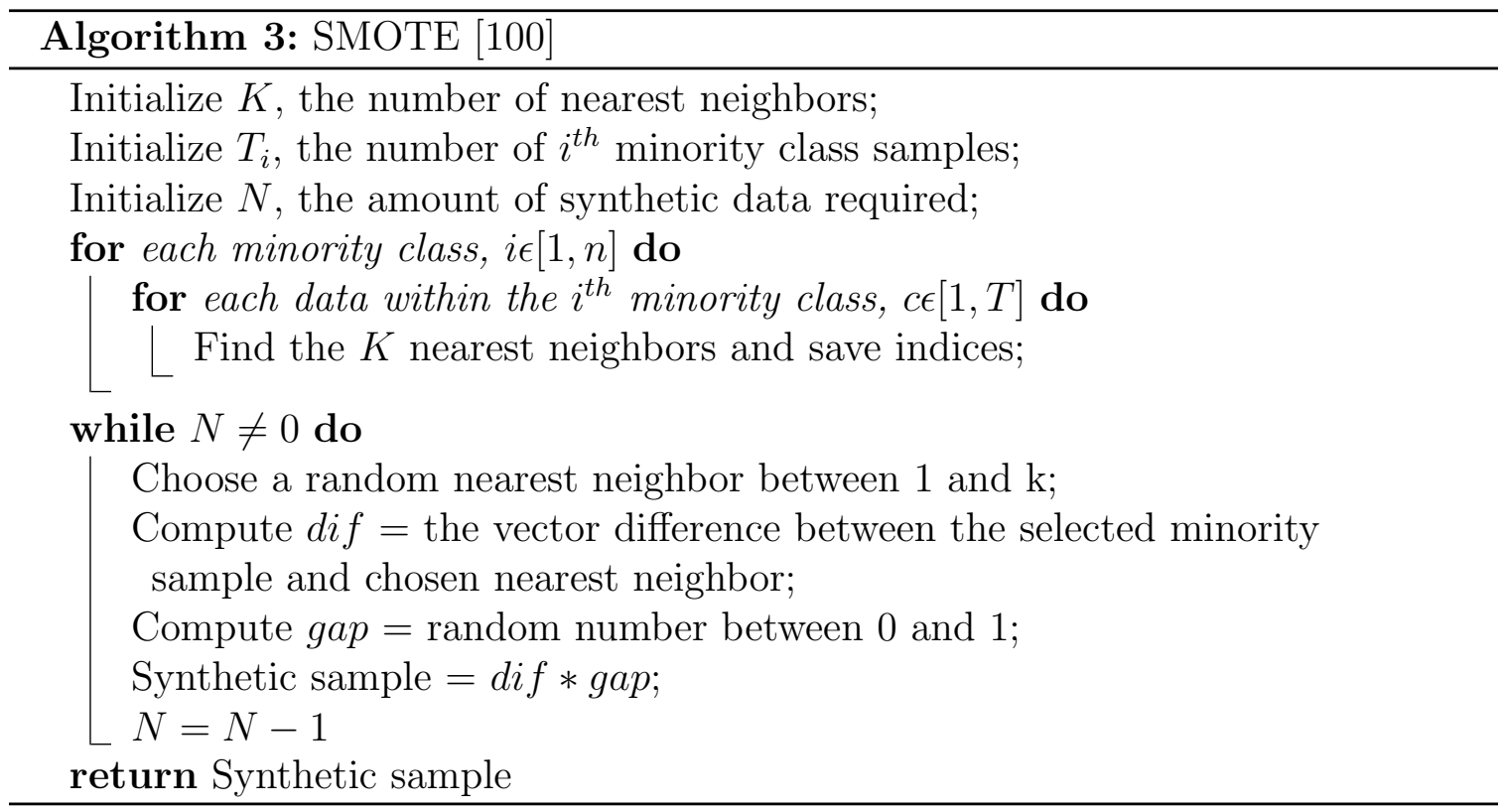

\section{NearMiss Undersampling}

On the other hand, under-sampling techniques focuses on reducing the size of majority samples. In NSL-KDD dataset, we set NORMAL and DOS as the majority classes since they take up to $53.46 \%$ and $36.46 \%$ of the whole dataset, respectively. The NearMiss [99] [98] under-sampling technique aims on the distance measures between these majority classes and a few specific minority samples. Two types of NearMiss approach are presented here:

NearMiss 1: Keep only the majority samples for which has smallest average distance to the $N$ nearest minority samples.

NearMiss 2: Keep only the majority samples for which has smallest average distance to the $N$ farthest minority samples.

After pre-processing the training data, we can put it into the RL structure. 


\subsubsection{Main Algorithm}

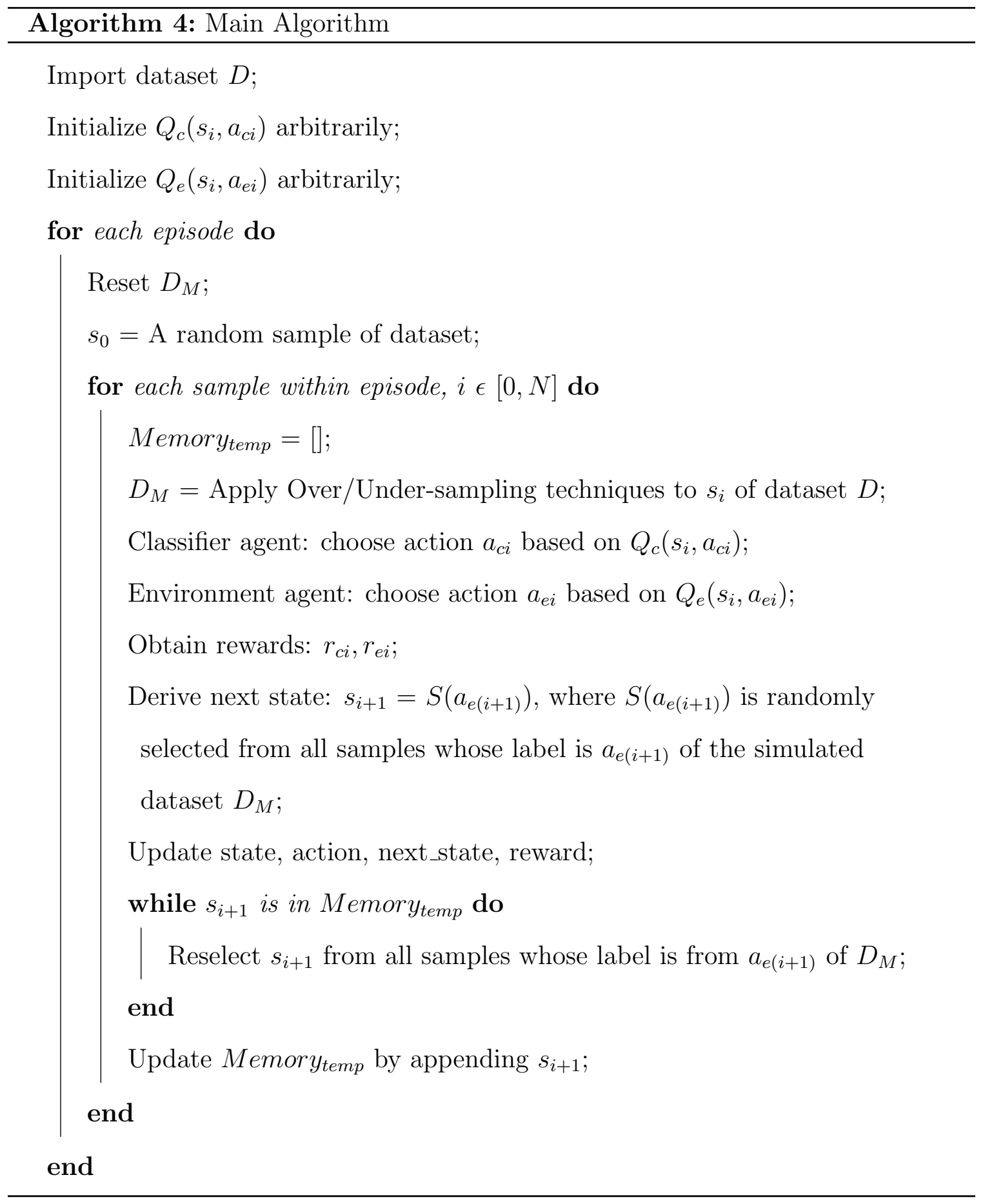

In addition to AE-RL, our algorithm provides a synthetic, more balanced, and more packed dataset before any learning process. Moreover, some features are added 
into the main algorithm for a more data-specific analysis. Every step learning is based on episodes and the number of episodes is based on the computation power of the machine. The training dataset, $D$, is modified by performing over/under-sampling techniques. A feature-selection is applied during the process, in which keeping the important characteristics of the newly generated samples in order to reduce biases. The modified dataset, $D_{M}$ is constantly being reset at the start of every episode so that brand new simulated datasets are generated and used for training the agent's adaptive skills.

Referring to Algorithm 2, the main algorithm starts by importing the original dataset. The $\mathrm{Q}$ values for the current data with the prediction of classes based on an accumulated policy, defined as $Q_{c}\left(s_{i}, a_{c i}\right)$ and $Q_{e}\left(s_{i}, a_{e i}\right)$, respectively, are arbitrarily initialized as they will eventually be auto adjusted by the RL process. For every episode, a random data is selected as the initial state before the RL process. Then, for every incoming data, the dataset is consistently being updated by performing over/under-sampling techniques to $s_{i}$, the current data's sub-class (eg. normal, back, ipsweep, ftp_write, and buffer_overflow) in keeping the ratio to its major class(eg. NORMAL, DOS, PROBE, R2L, and U2R). Next, RL components are discovered for both classifier and environment agents. Following their given rewards, classifier will make prediction of the current class type, $a_{c i}$, based on its policy, $Q_{c}(s, a)$, while environment provides the next training data, $a_{e i}$, based on previous classifier's performance, $Q_{e}(s, a)$. In the case of NSL-KDD, $a_{c i}$ is an element of NORMAL, DOS, PROBE, R2L, and U2R and $a_{e i}$ is an element of the features in the training dataset. Similarly, $r_{c i}$ is the positive or negative reward corresponding to classifier's correct or incorrect classification, respectively. Meanwhile, $r_{e i}$ takes opposite rewards as the classifier agent. The next state, $s_{i+1}$, is derived by randomly picking a sample from $a_{e(i+1)}$, the resulting class decided by the environment agent. Meanwhile, a 
memory checker is constructed in parallel to eliminate the existence of duplicated data during the data selection phase.

The algorithm progresses along with a decreasing epsilon-greedy policy. Both agents start off with a high exploration rate, $\epsilon=1$, indicating the fact that they had no previous knowledge of the dataset. Then, their rates gradually decreases as the learning goes on and converges to lower bounds of $\epsilon_{c}=0.01$ and $\epsilon_{e}=0.8$, for the classifier agent and environment agent, respectively. $\epsilon_{c}$ is set relatively low since an ideal predictability for the classifier agent is to have the entire control of the training set based on existing knowledge. On the other hand, the environment agent requires a more arbitrary distribution method of data in cases of uncontrollable learning patterns from the classifier agent.

\subsubsection{Models and Error Reduction}

Let's recall that Bellman equation relating to $\mathrm{Q}$ functions of consecutive steps is:

$$
Q^{*}(s, a)=R(s, a)+\gamma \max _{a^{\prime}} Q\left(s^{\prime}, a^{\prime}\right)[63]
$$

Through a temporal difference learning which iteratively approximates the $\mathrm{Q}$ values at each time step, a loss function can be derived so that we can minimize the mean squared error for more accurate estimations:

$$
L=\frac{1}{2}\left[R(s, a)+\gamma \max _{a^{\prime}} Q\left(s^{\prime}, a^{\prime}\right)-Q\left(s^{\prime}, a^{\prime}\right)\right][63]
$$

Since we can never be accurate in estimating the actual $Q^{*}(s, a)$, in practice, we apply the gradient descent for every iteration to find the local minimum of loss and eventually arrives at the global minimum of the loss function $L$. In addition, we use Huber loss as the base for the actual loss function to apply robust regression, which 
consists of a piecewise function that is quadratic when the parameter is small and becomes linear beyond a threshold. The advantage is that it eliminates sensitivity to outliers (i.e., explosive behaviours).

\section{Double Deep Q-Network}

Consider the Q-function, more specifically:

$$
\max _{a^{\prime}} Q\left(s^{\prime}, a^{\prime}\right)
$$

Taking the maximum Q-value among all possible actions at a given state is our current standard for getting the optimal value of the learning process. However, this would cause an overestimation of the actual Q-values. For instance, suppose the true label for a specific data is R2L, if the decision-changing features are distributed some above and some below the decision threshold, by taking the maximum of these estimates would always lead to the choice of one above the threshold.

To deal with this concern, we choose the Double Deep Q-Network (DDQN) for our model structure. The DDQN structure involves using two separate Q-value estimators for action-value estimation. They function as constraints to each other by selecting actions using opposite estimators. Thus, the maximization bias can be disentangled.

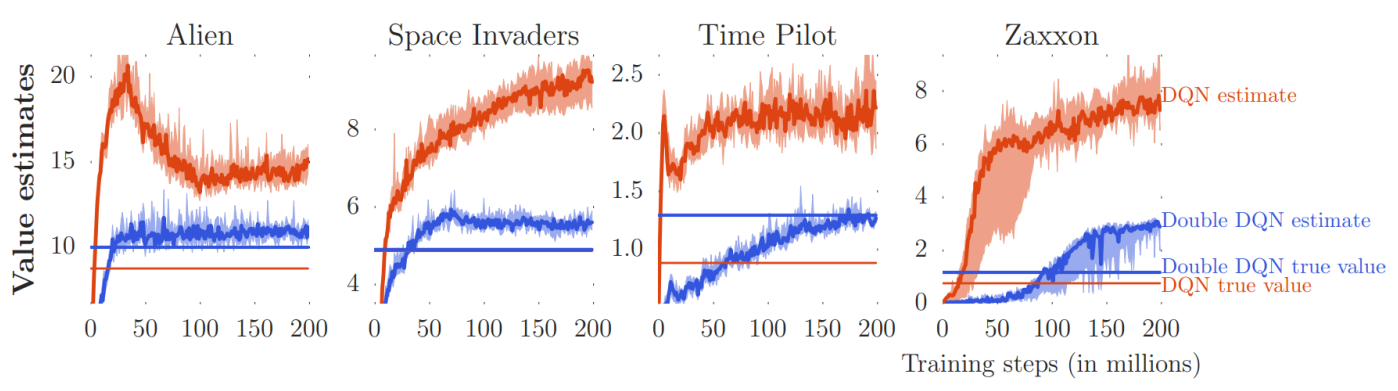

Figure 3.1: Overestimates of Q-learning

[107] 
As we can see from Figure 3.1, the value estimates by DQN (orange) tend to significantly overestimate the action-values comparing to the value estimates by DDQN (blue).

In this dissertation, the details of DDQN algorithm we used is as follows:

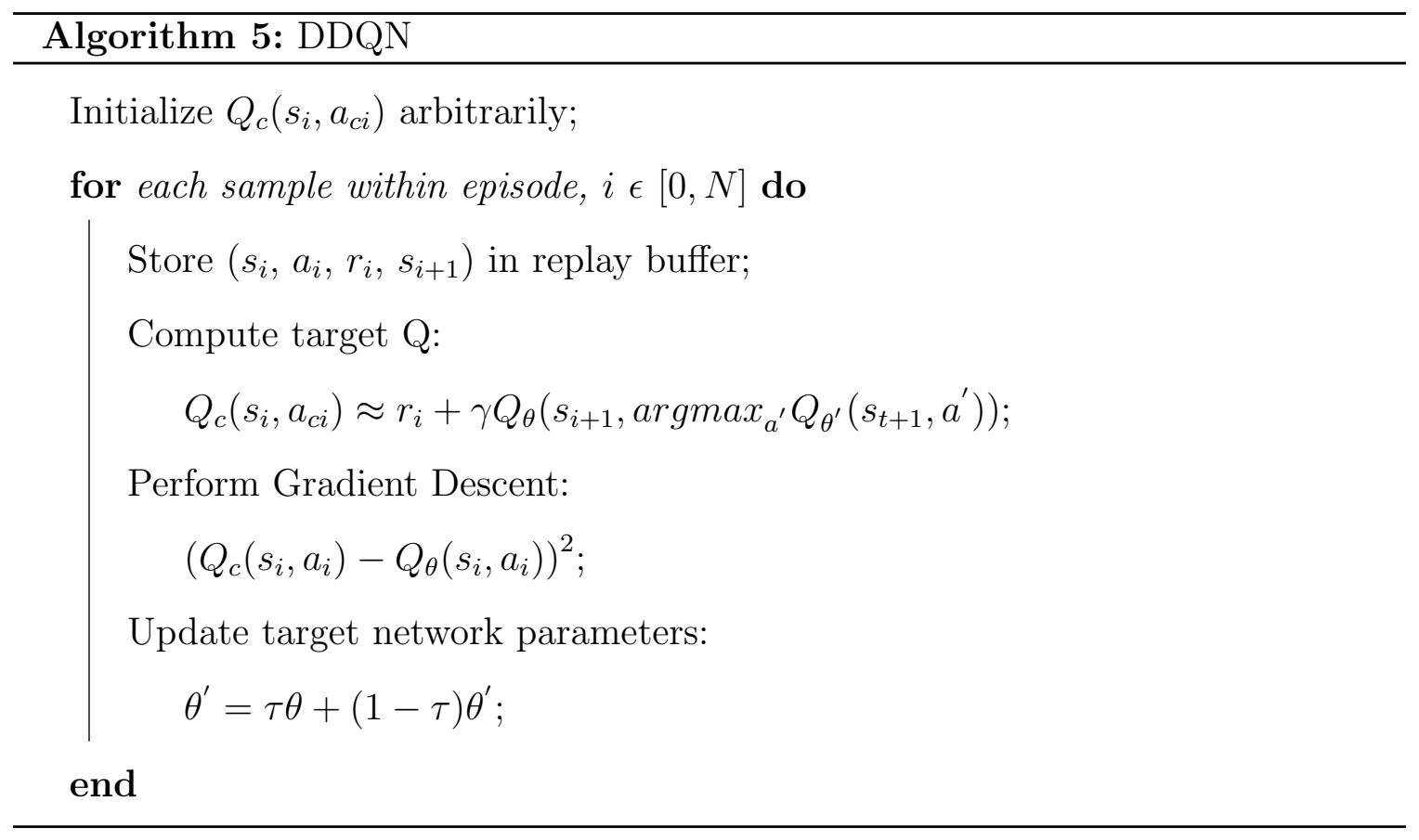

After a random initialization of $Q_{c}\left(s, a_{c}\right)$, for each incoming data, the Q-values are being updated. As opposed to a regular DQN, where actions are chosen by the innate Q-learning policy, DDQN always chooses the optimal action based on the $Q_{\theta^{\prime}}$ function, which is a function approximation. Then, we minimize the mean squared error between $Q_{c}\left(s, a_{c}\right)$ and $Q_{\theta^{\prime}}$ for a better action evaluation. Lastly, $Q_{\theta^{\prime}}$ is slowly being transformed to $Q_{c}\left(s, a_{c}\right)$ through Polyak averaging, where $\theta^{\prime}$ refers to the target network parameter, $\theta$ refers to the primary network parameter and $\tau$ refers to the rate of averaging which is set to 0.01 as a default hyper-parameter [108]. 


\subsubsection{Algorithm Overview}

Figure 3.2 demonstrates a detailed procedure of our algorithm, which concludes all parts mentioned above. Now, let's go through the algorithm with one simulation trial. Assume a majority class sample, say normal (NORMAL), is selected as the initial state from the original training set $D$ and is being passed to the environment agent (EA). Without any previous knowledge about the data, EA makes a prediction, $a_{e i}$ (23 labels) based on an arbitrary $\mathrm{Q}$ function, $Q_{e}\left(s_{i}, a_{e i}\right)$, say neptune (DOS) and on one hand performs over-sampling/under-sampling techniques (OS/US) to produce a modified dataset, $D_{M}$ and the other passes into the RL environment for modelling. Then, a neptune sample from $D_{M}$ is randomly selected and passed to both the EA and classifier agent(CA) as the next state. Meanwhile, Q functions are updated for both EA and CA by going through a series of modelling with DDQN, gradient descent, and loss reduction in the RL environment. Receiving the state and updated Q functions, EA and CA could consistently take actions for every incoming data. At the end of every episode, $D_{M}$ will be reset to the original training set, where the dynamic training occurs by training a dataset that had new data samples added dynamically. After training the specified total number of episode, CA makes prediction of the final classes (5 labels) on the testing set. 


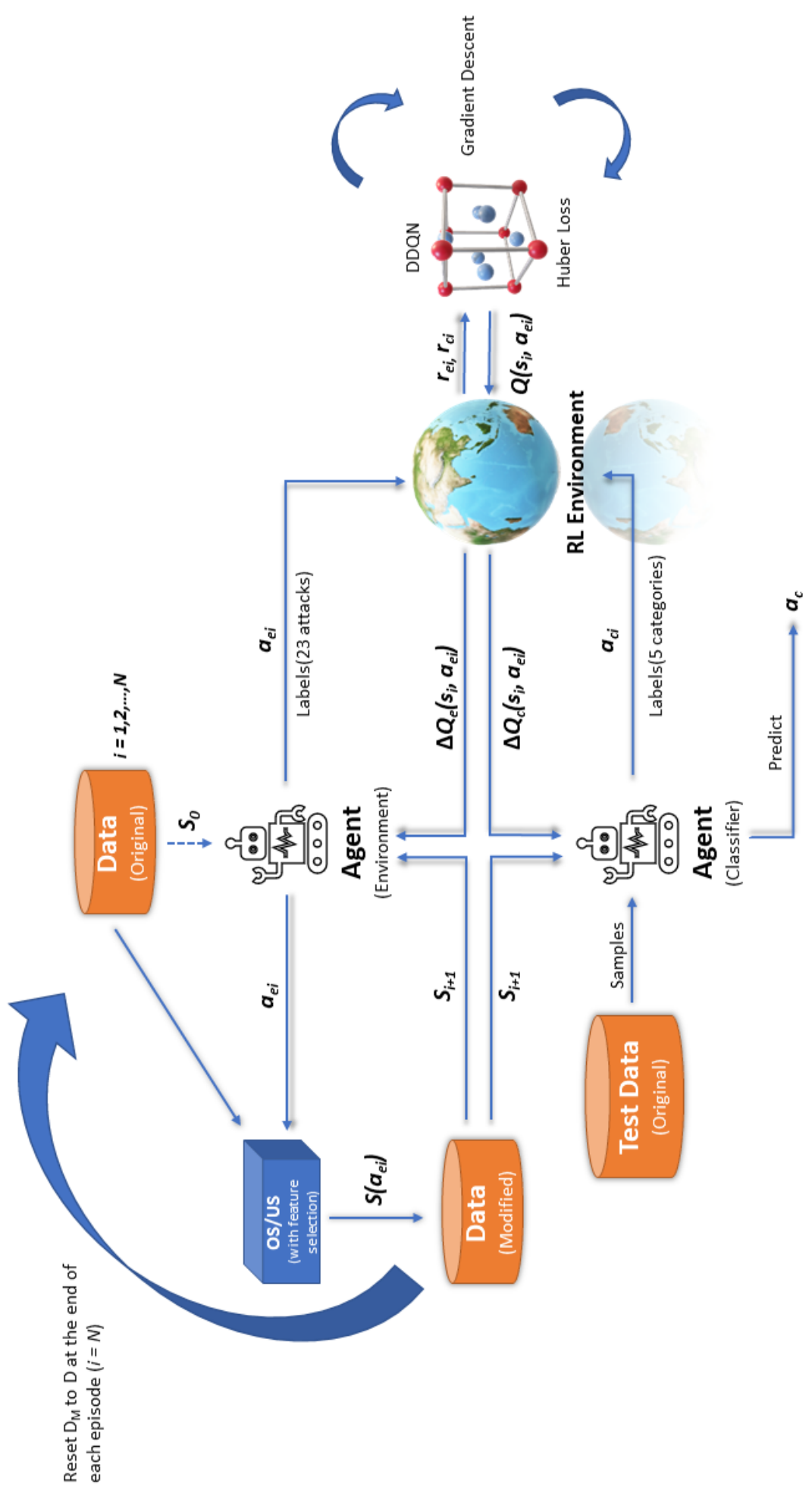

Figure 3.2: Algorithm Structure 


\section{Chapter 4}

\section{Evaluation and Result Analysis}

\subsection{Dataset Description}

As mentioned before, the dataset is split into a training set of 125973 samples and a testing set of 22544 samples. The datasets contain 32 continuous, 6 binary, and 3 nominal features. In order for agent to perform better training in an organized manner, the datasets are further transformed. The continuous features are scaled down to a range between 0 and 1 while the nominal features are one-hot encoded into dummy variables. Hence, the datasets are finalized to 122 features with 32 continuous and 90 binary. When comparing the training and testing set, a few facts may become a concern:

1. Both datasets have only 21 labels in common.

2. 2 labels are unique that only appear in the training set and 17 labels are unique that only appear in the testing set.

Hence, this implies that a lot of testing cases are not being trained at all in the training set, which would be a challenge as the agent has to be accurate enough to generalize the categories in order to do well against the untouched samples. However, this problem can be solved as follows. 


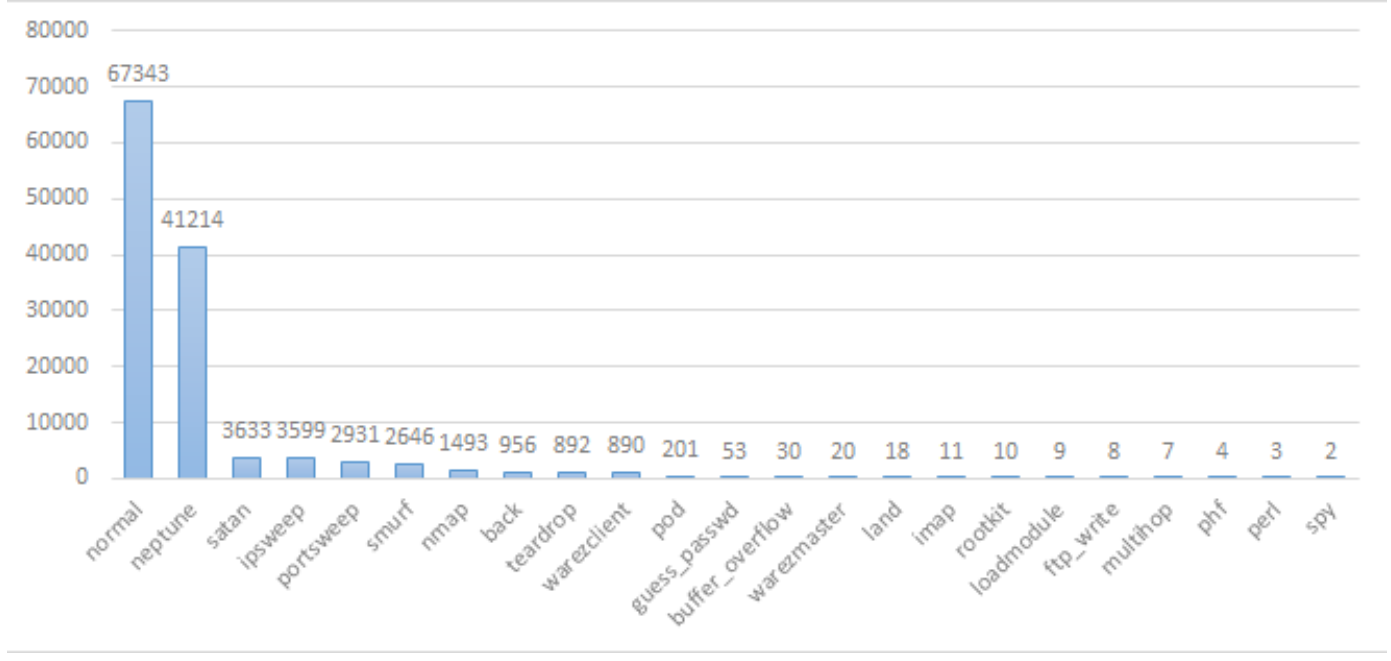

Figure 4.1: Subclasses

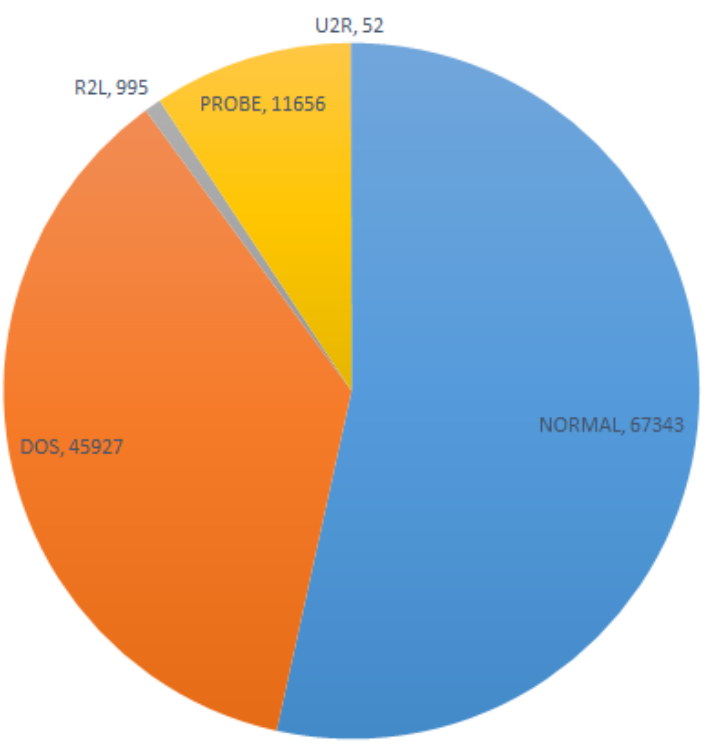

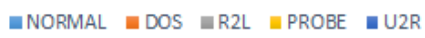

Figure 4.2: Distribution of Categories 
As Figure 4.1 demonstrated, the subclasses normal and neptune takes the majority portion of the dataset, $53.45 \%$ and $32.71 \%$, respectively, whereas the remaining subclasses only take $13.84 \%$. Thus, the dataset is extremely unbalanced.

Now, to make the dataset meaningful, these 23 labels are categorized into five categories: NORMAL, DOS, PROBE, R2L, and U2R [105]. (Further details can be found in Table 3.1) The advantage of this action is that even for the new samples in the test set, the agent is able to predict its category, rather its subclasses.

As Figure 4.2 demonstrated, the imbalanced property becomes more evident as the biggest class NORMAL contains $53.45 \%$ while the smallest class U2R only contains $0.04 \%$ of the whole dataset.

Figure 4.3 displays the architecture of input and output on which both agents are based. In the architecture, two neural networks are implemented with shallow layer numbers of 3 and 5 for environmental and classifier agent, respectively. The input of both agents are equivalent as they take in all the features in the training data for learning. Their outputs, however, are different as they serve for different purposes. The objective for environmental agent is to summarize the most needed samples for training at the moment, which requires the output to be as specific as it can. Hence, the output of environmental is the 23 labels. On the other hand, classifier's objective is to produce a meaningful prediction of attacks so that intrusion defender can take immediate actions. As mentioned previously, data is categorized into 5 categories according their natures. Hence, the output of classifier agent is the 5 categories. Another advantage of this different output strategy is that it has the ability to cope with the untrained labels. The classifier agent would always make the most optimal estimation based on its learned characteristics of closest category. 


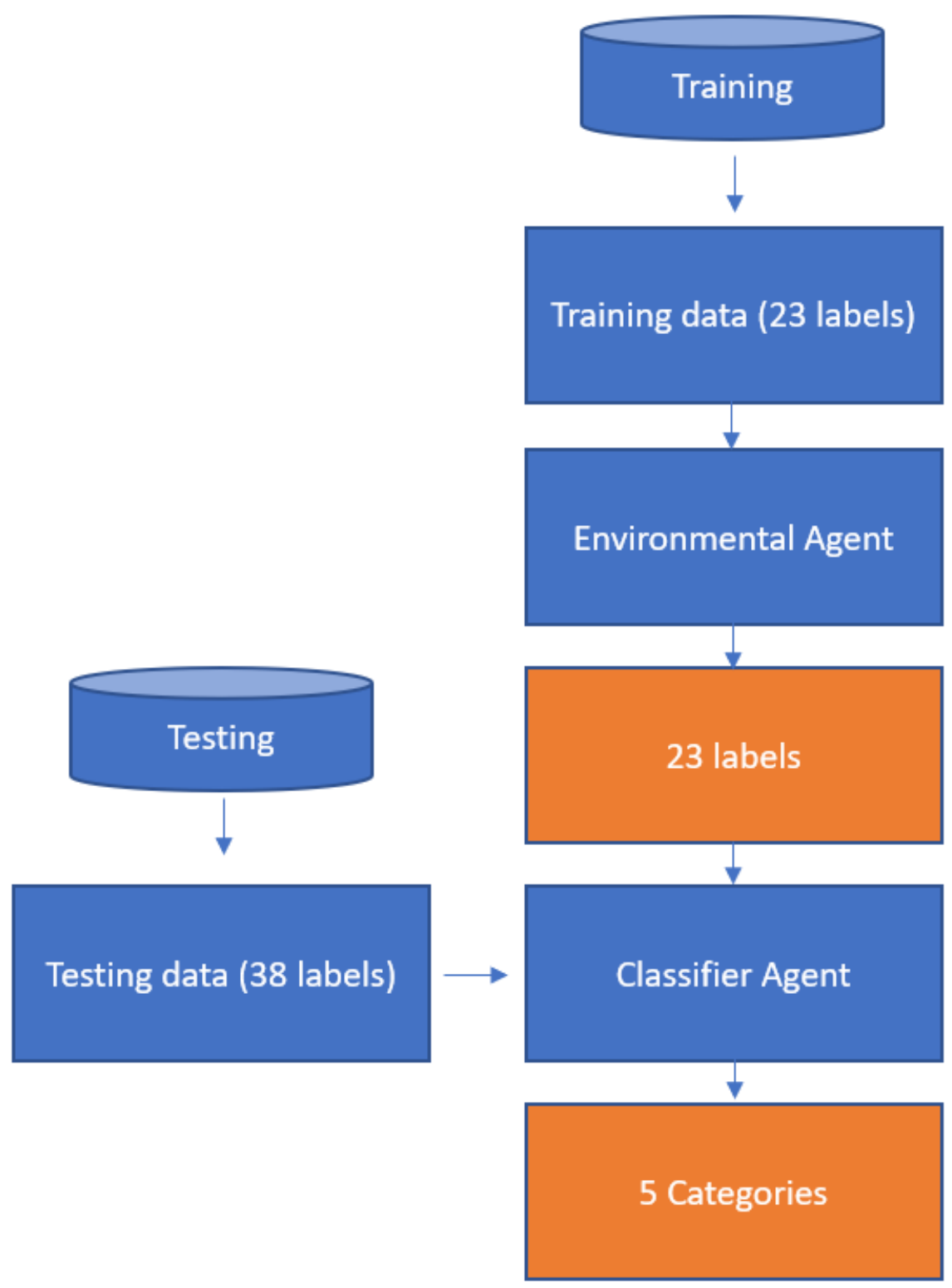

Figure 4.3: Input and Output 


\subsection{Evaluation Metrics}

When evaluating the performance of the prediction, two evaluation metrics are used: classification accuracy and F1-score. The definitions are as follows:

Accuracy is the most intuitive performance measure as it is simply the ratio of correctly predicted observations to the total observations. Yet, one shortage of using accuracy as measurement is that it is only good when the false positive and false negative rates are relatively close to each other. Otherwise, it should only be considered as a reference parameter.

$$
\text { Accuracy }=\frac{\text { Number of correct predictions }}{\text { Total number of predictions made }}[109]
$$

Precision is the ratio of correctly predicted positive observations to the total predicted positive observations. Hence, a high precision rate indicates a low false negative rate, which can act as a rigorous measure to limit overestimations.

$$
\text { Precision }=\frac{\text { True Positives }}{\text { True Positives }+ \text { False Positives }}=\frac{\text { True Positives }}{\text { Total Predicted Positives }}[109]
$$

Recall is the ratio of correctly predicted positive observations to the total positive obervations. This measurement is used to check the prediction is accurate enough for the positive class.

$$
\text { Recall }=\frac{\text { True Positives }}{\text { True Positives }+ \text { False Negatives }}=\frac{\text { True Positives }}{\text { Total Actual Positives }}[109]
$$

F1-score is the combined calculation of precision and recall. By taking the weighted average of both measurements, it takes both false positives and false negatives into account. Hence, it is more reliable to use for imbalance datasets.

$$
\text { F1-score }=2 * \frac{1}{\frac{1}{\text { precision }}+\frac{1}{\text { recall }}}[109]
$$




\subsection{Tools}

In pursuance of loading the datasets, transferring datasets, building the RL framework, calculating loss functions, evaluating the performance and visualizing the results, a variety range of tools are used. Beyond the original AE-RL algorithm [1], we also have added a few libraries for performing sampling techniques. After reviewing all the tools for implementation, several important ones are displayed as follows:

\section{Tensorflow}

A computing graph processor which is used for constructing neural network for Qfunction and Huber losses [110]. Examples: tf.where(), tf.select.

\section{Keras}

An open-source neural-network library in Python that integrates well with Tensorflow operations [111]. Examples: keras.models, keras.layers.

\section{Pandas}

An open-source, powerful, flexible, data analysis tool, mainly used for organizing the training data's dataframe [112]. Examples: pd.read_csv(), pd.concat().

\section{NumPy}

A Python library used for supporting large, multi-dimensional arrays, and matrices, mainly for the formatted training dataset [112]. Examples: np.random(), np.sum(). 


\section{Scikit-learn}

A machine learning library that features in various classification, clustering and regression algorithms, mainly used for generating metrics in this dissertation [113]. Examples: sklearn.metrics, sklearn.utils.

\section{Imblearn}

A Python library used for performing over-sampling and under-sampling techniques on an imbalanced dataset [100]. Examples: imblearn.over_sampling, imblearn.under_sampling.

\section{Time}

A built-in Python module used for recording the run time of the code. Examples: time.time().

\section{Random}

A built-in Python module used for generating random numbers within a given range. In this dissertation, since only part of the training data is being trained and some random sampling is required after the over-sampling process. Examples: random.randint(). 


\section{Json}

A built-in Python module used for storing and exchanging data. In this dissertation, the trained model is stored as json to pass into the testing file. Examples: json.dump () .

\section{Matplotlib}

A plotting Python library used for generating plots and have close relationships to NumPy and Pandas. In this dissertation, it is mainly used for performance evaluation such as visualizing accuracy, precision, reacall, and F1-score. Examples: plt.plot(), plt.title () .

\subsection{Parameter Setup}

When performing the training, several major parameters had been examined and determined to be certain values which is ideal for the model. As training begins, the exploration rates, $\epsilon$, are set to lower bounds of 0.01 and 0.8 for the classifier agent and the environmental agent, respectively. Since $\epsilon_{c}$ is used to progress the classification tasks, hence it is strictly following a regular bound. On the other hand, $\epsilon_{e}$ is used to choose tasks for training, hence as long as the distribution is relatively balanced, it is encouraged to be more explorable. During the Q-learning, the learning rates for both agents are set to 0.2 and the discount factor is set to 0.001 . The layers of the neural network, $l_{c}$ abd $l_{e}$ are set to 5 and 3 for the classifier and environmental agent, respectively. The number of nearest-neighbors that is used to generate synthetic data, $k$, is set to a value greater equal to 3 . Since several subclasses have a small quantity of samples, such as multihop (7), ftp_write (8), loadmodule (9), imap (11), and land (18), a large number of nearest-neighbors would produce bias results. For cases such 
Table 4.1: Training Parameters

\begin{tabular}{cl}
\hline Parameter & Description \\
\hline$\epsilon_{c}$ & Exploration rate for classifier agent, $>0.01$ \\
\hline$\epsilon_{e}$ & Exploration rate for environment agent, $>0.8$ \\
\hline$\alpha_{c}$ & Learning rate for classifier agent, $=0.2$ \\
\hline$\alpha_{e}$ & Learning rate for environment agent, $=0.2$ \\
\hline$\gamma$ & Discount factor, $=0.001$ \\
\hline$l_{c}$ & Hidden layers for classifier agent's neural network, $=5$ \\
\hline$l_{e}$ & Hidden layers for environment agent's neural network, $=3$ \\
\hline$k$ & Number of Nearest-Neighbors for SMOTE, $\geq 3$ \\
\hline num_episodes & Number of training episodes, $\geq 100$ \\
\hline iterations_episode & Number of iterations per training episode, $\geq 100$ \\
\hline
\end{tabular}

as spy (2), perl (3), and phf (4), we select $k$ as 1 . Through repeatedly testing, we set the number of training episodes and iterations per episode lower bounds of 100 since the performance is reaching its optimal after these values.

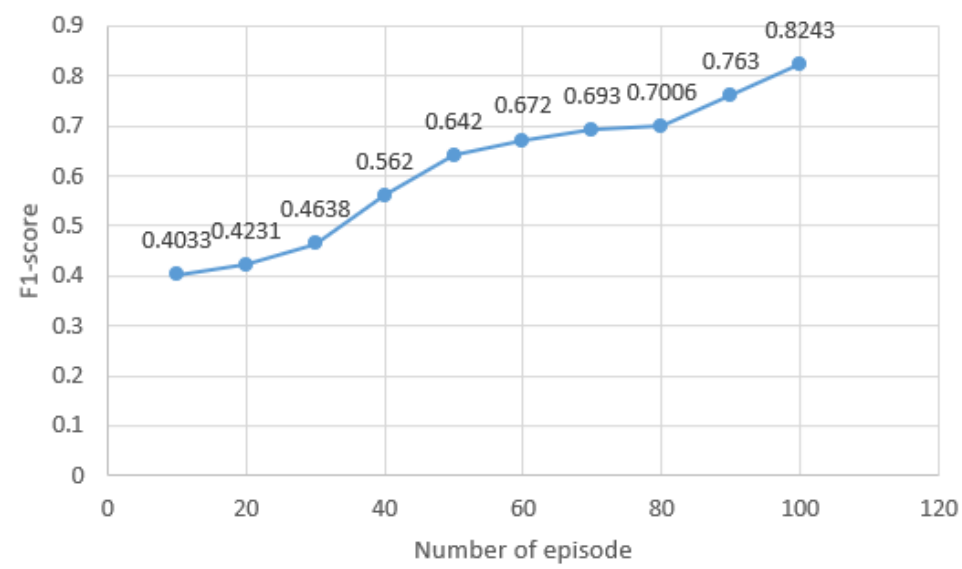

Figure 4.4: F1 scores for different number of episodes

As demonstrated in Figure 4.4 and Figure 4.5, we observe that both F1 and accuracy have an increasing trend from 10 episodes to 100 episodes. The performance 


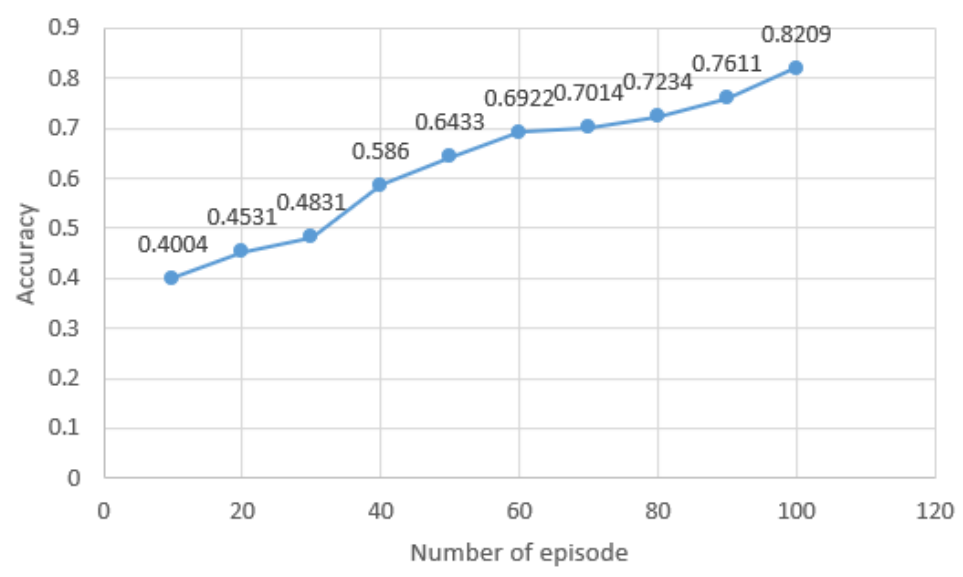

Figure 4.5: Accuracy scores for different number of episodes

after 100 episodes becomes stable around 0.82 for both F1 and accuracy. Hence, the number of episode for training is tuned to be greater than 100 episodes to achieve best results.

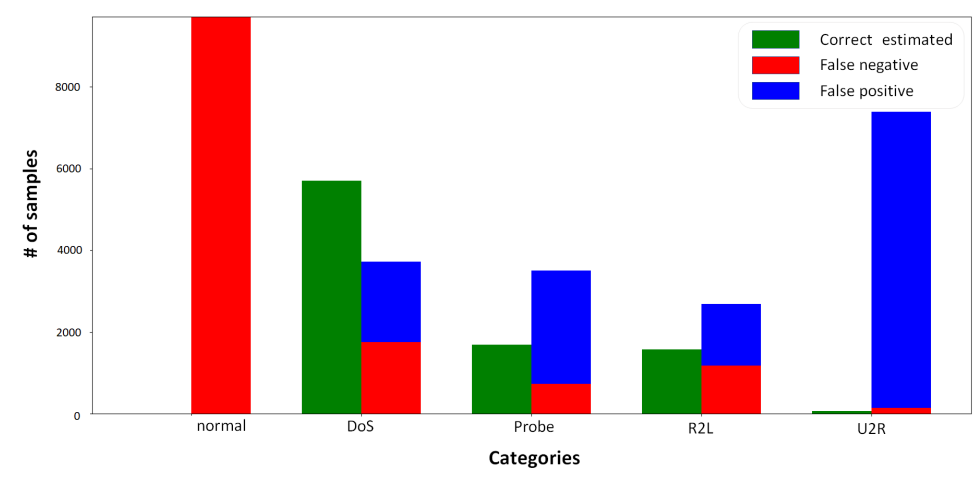

Figure 4.6: F1 score: 0.4033 for 10 episodes

Figure 4.6, 4.7, and 4.8 display the changes as number of episodes increase. At 10 episode, the class U2R is being overestimated. This is due to the fact that we forced the balanced learning strategy onto the classifier agent and it's not mature enough to identify the differences between classes yet. However, it is showing a sign that our mechanism starts to kick in as the agent is treating all classes equivalently. At 50 episode, the agent is already mature enough to clearly identify NORMAL class, 


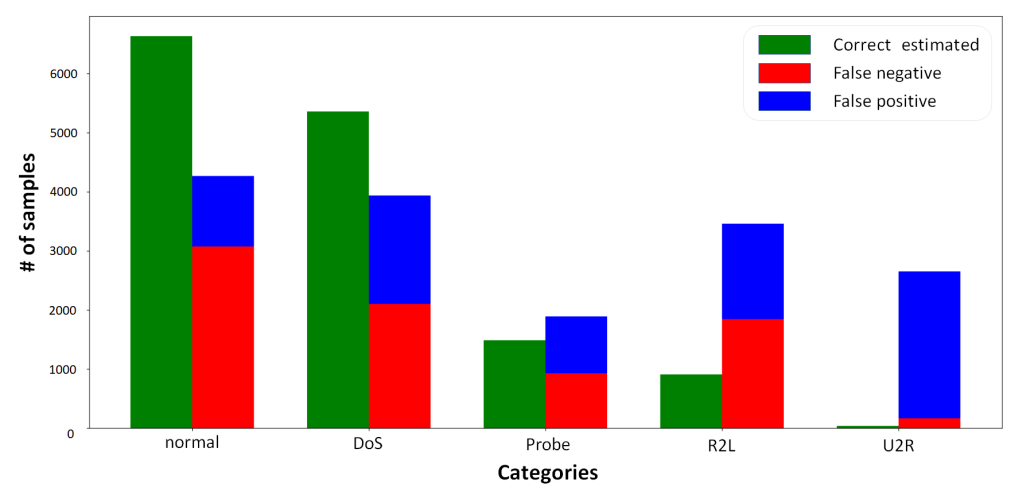

Figure 4.7: F1 score: 0.642 for 50 episodes

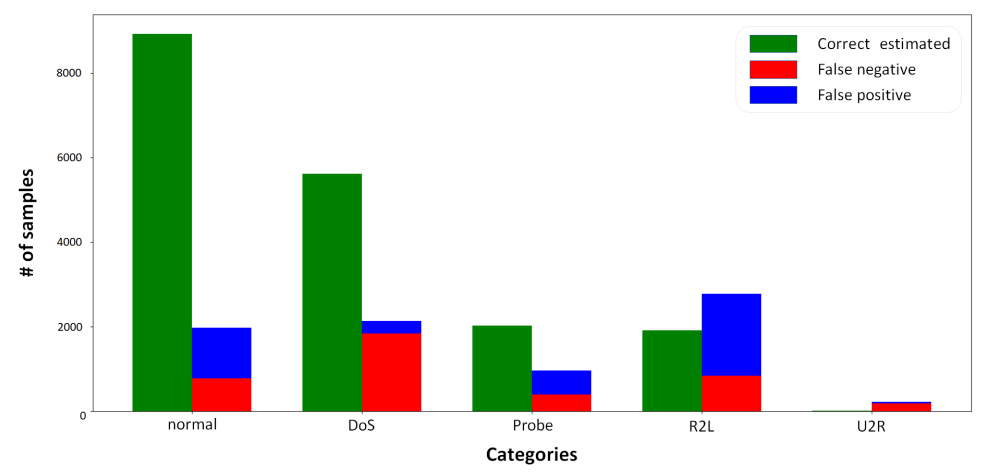

Figure 4.8: F1 score: 0.824 for 100 episodes

reaching a descent true positive rate, yet still unclear on the minority parts. Finally, at 100 episode, the boundary between the classes is becoming more clear and achieves an F1 of 0.824 . 


\subsection{SMOTE Performance}

In this section, we are going to demonstrate the performance of SMOTE on the AERL framework. As note, the purpose of SMOTE is to generate synthetic samples in accordance to its own data patterns so that minority classes have enough samples to be transferred to the classifier agent for training. Considering the highly unbalanced property of the dataset, the metric F1 will be used to measure performance [114]. As mentioned before, F1 takes both precision and recall into consideration, which assures both false-positive and false-negative into account. In our case, false-positive and false-negative rates are both crucial measures. For instance, a NORMAL class is predicted to be an intrusion attack, say $D_{o S}$, can lead to false-alarm. F1-score can be further divided down to "one vs. rest" and "aggregated". Considering the "one vs. rest" approach, the NORMAL class is the "one" while all other four classes belong to the "rest", which results in a binary classification. On the other hand, the "aggregated" refers to all classes being equivalent. In this dissertation, we use the aggregated F1-score as our measure, which takes the weighted average of all F1-scores [113]. For result comparison, we are interested in the F1-score trending on SMOTE performance.

Equal amount of samples are generated for both minority classes U2R and R2L. For instance, if we generate 20000 samples, 10000 samples will be generated for $\mathrm{U} 2 \mathrm{R}$ and R2L, respectively. To test the trending, 20 trials had been recorded for presentation. As shown in Figure 4.9, we can observe that the algorithm AESMOTE's performance, although fluctuating, but demonstrating an increment, reaching a peak of $\mathrm{F} 1=0.8243$, when the sample size is between 5000 to 70000 . After a closer inspection of the result data, we observe that the implementation of SMOTE had putting on some negative effects at first, causing the F1-score averaging around 0.7 while the original AE-RL has an average of 0.78 . This is due to the instability of 


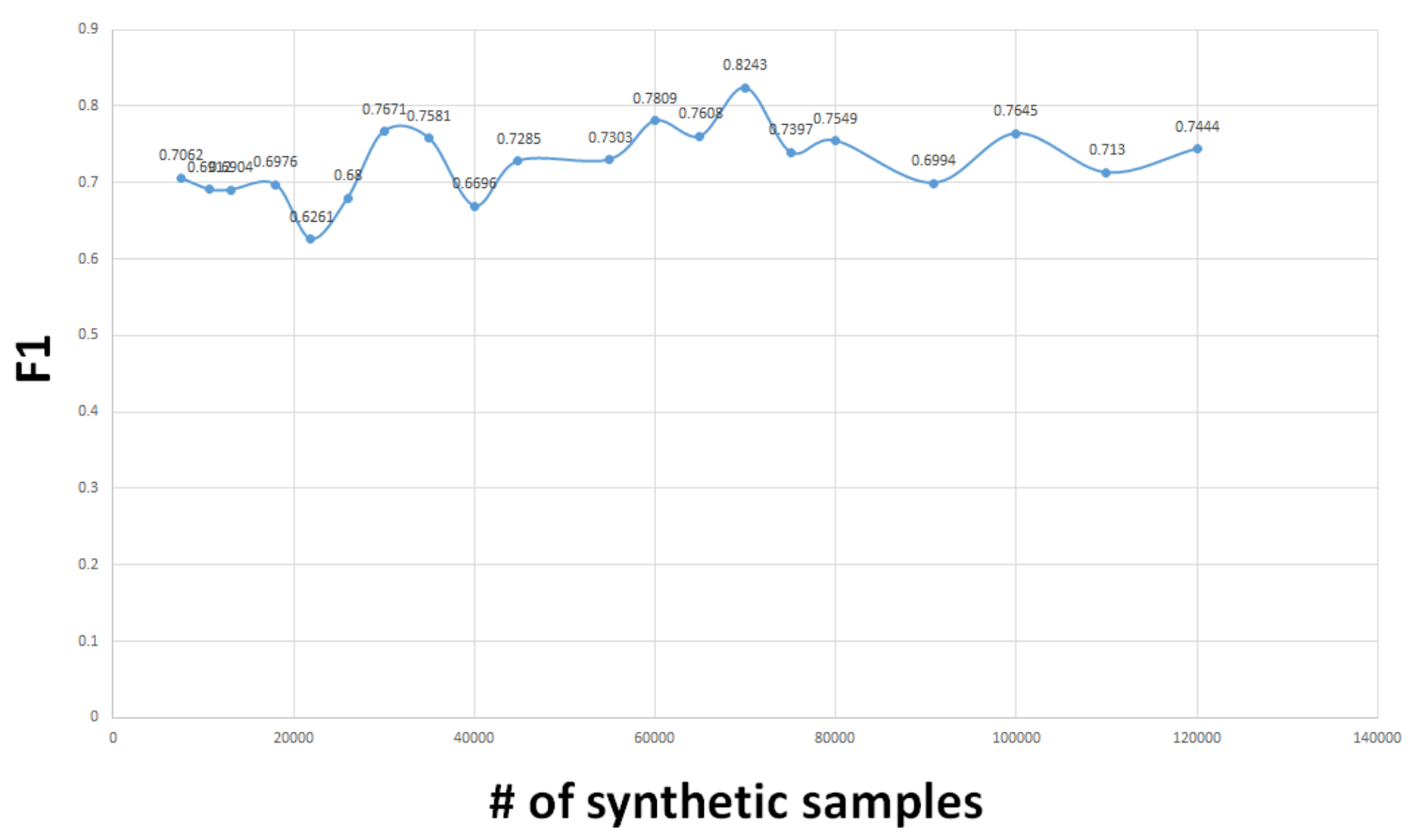

Figure 4.9: F1-score Trending on SMOTE

newly generated samples. Although, theoretically, the synthetic samples are "within" its neighbours, but they are still not as reliable since they might produce new nonrealistic pattern which could affect the real data's performance. However, as more samples are being generated, this non-realistic pattern becomes stable enough and slowly separates itself from the real data's pattern. The advantage of this newly created pattern is that it could become useful to deal with the non-trained labels in the testing set. This is also the reason that an outstanding score of 0.825 could be found in the trend as opposed to the flat average of 0.78 by AE-RL.

Looking at the confusion matrix for both algorithms in Figure 4.10 and Figure 4.11, we observe that the true-positive rate for the minority class R2L has a significant increase from 0.29 to 0.69 , showing an improvement due to the over-sampling mechanism. As we look into the subclasses in Figure 4.12 and Figure 4.13, we can see that the number of correct estimates for the minority class R2L has a significant increase for AESMOTE comparing against the original AR-RL. 


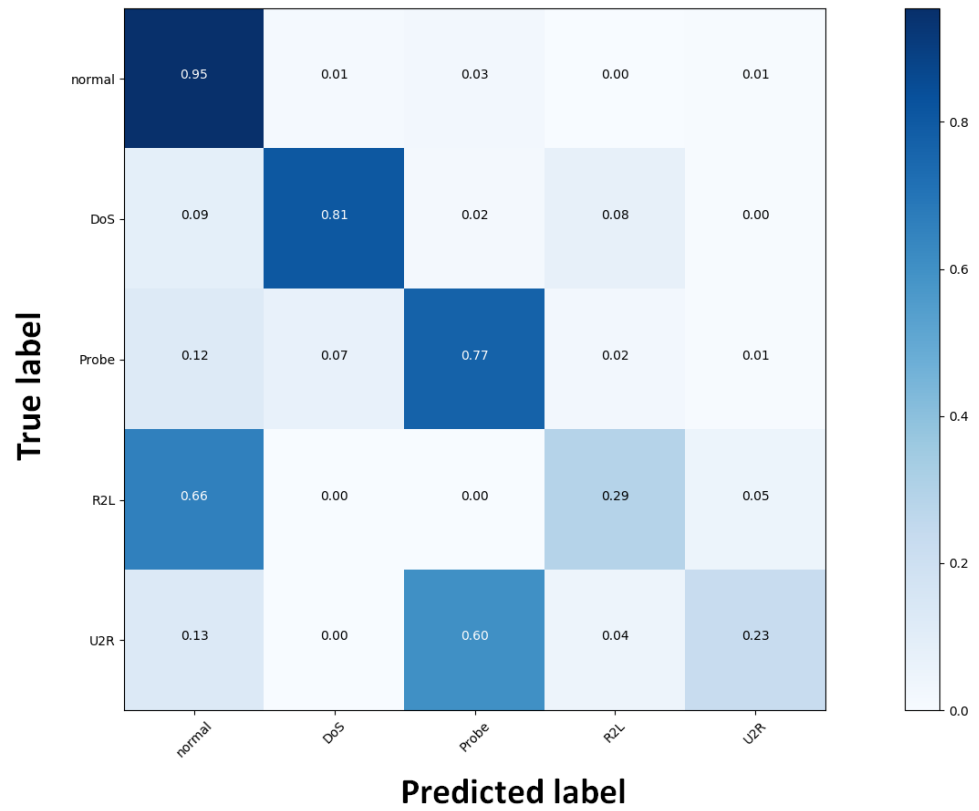

Figure 4.10: Confusion Matrix for AE-RL

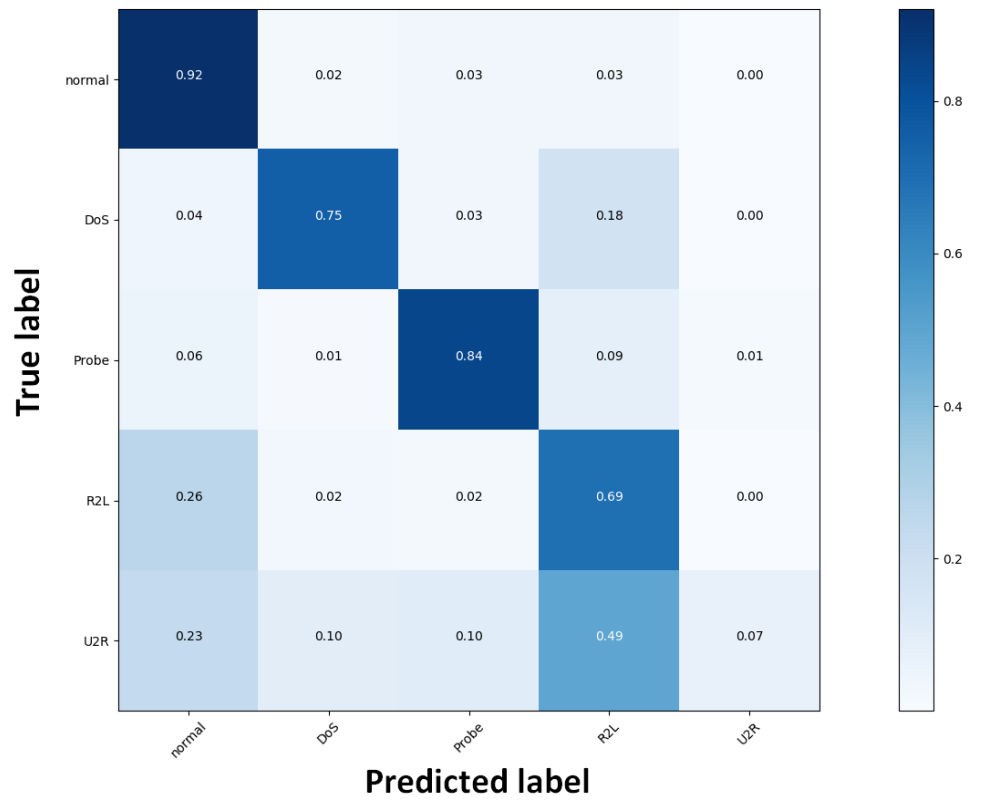

Figure 4.11: Confusion Matrix for 70000 samples with SMOTE 


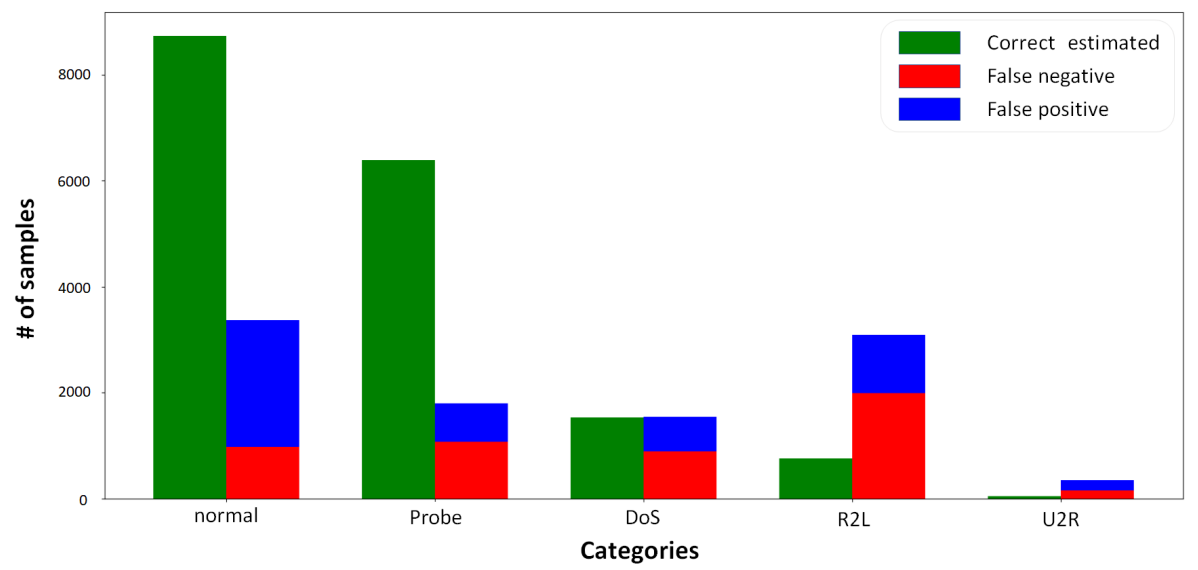

Figure 4.12: AE-RL, F1 $=0.78$

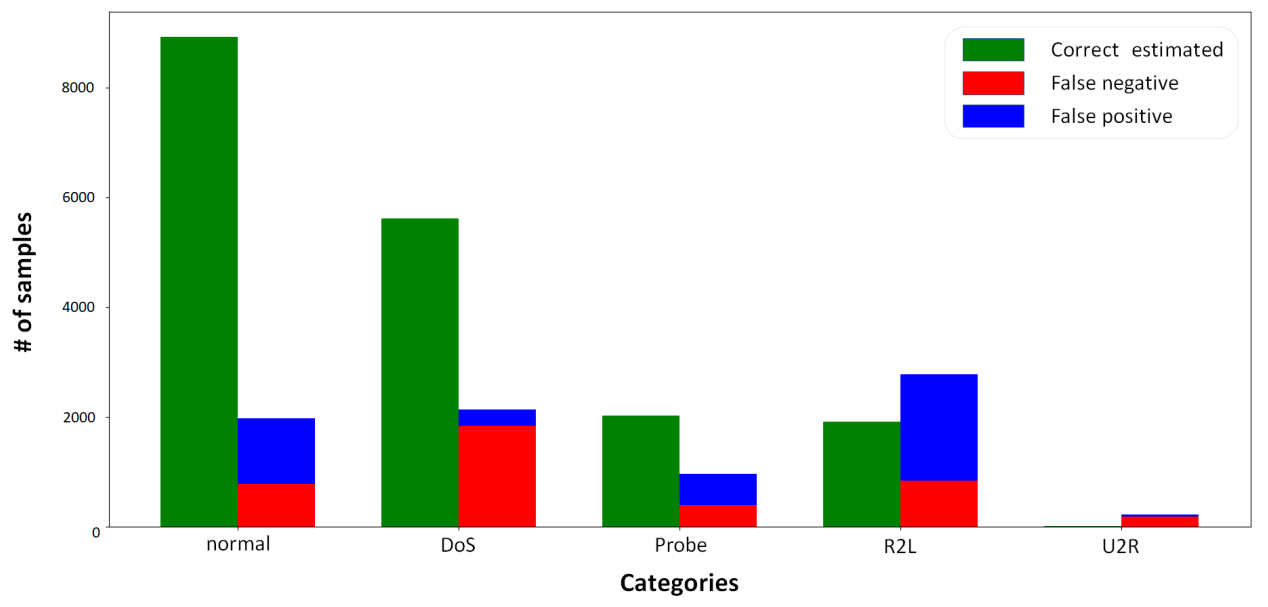

Figure 4.13: AESMOTE, F1 = 0.8243 


\subsection{Other Performance}

Other than the SMOTE sampling method, we've also tried implementing Random Over Sampling (ROS) [95], NearMiss1 and NearMiss2 [99] [98] under sampling method. Results are obtained as follows:

Table 4.2: Across Algorithms

\begin{tabular}{|l|l|l|l|l|l|}
\hline Method & F1-score & Accuracy & Precision & Recall & Runtime(sec.) \\
\hline AE-RL & 0.78 & 0.7744 & 0.7616 & 0.7744 & 350 \\
\hline AESMOTE & 0.8243 & 0.8209 & 0.8411 & 0.8209 & 2000 \\
\hline ROS & 0.6932 & 0.6977 & 0.7112 & 0.6932 & 1000 \\
\hline NearMiss1 & 0.7602 & 0.7611 & 0.7795 & 0.7611 & 195 \\
\hline NearMiss2 & 0.7865 & 0.7805 & 0.8027 & 0.7805 & 84 \\
\hline
\end{tabular}

As note, the main idea of ROS is duplicating existing data, which is against our initial purpose. After several trials, the highest F1-score that we obtained were 0.6932. Theoretically, this method is considered the most unreliable method, although it does have the potential of discovering new data patterns, its performance is relatively non-stable. For undersampling techniques such as NearMiss1 and NearMiss2, the NORMAL and DOS are considered as the majority classes. Yet again, this does not solve the duplicated data problem since it only applies a decrement of the majority classes' data size. However, they do have their own advantage of a faster running runtime of 195 seconds and 84 seconds, respectively and only 10 episodes are required for training due to the data size reduction. Comparing to AE-RL, AESMOTE has a longer runtime due to the addition of sampling techniques and feature-selection mechanism, yet they have brought up the F1-score to 0.8243 .

Figure 4.14, 4.15, and 4.16 shows the detailed prediction of each class. We can observe that all of them have weak performances on minority classes with low true 


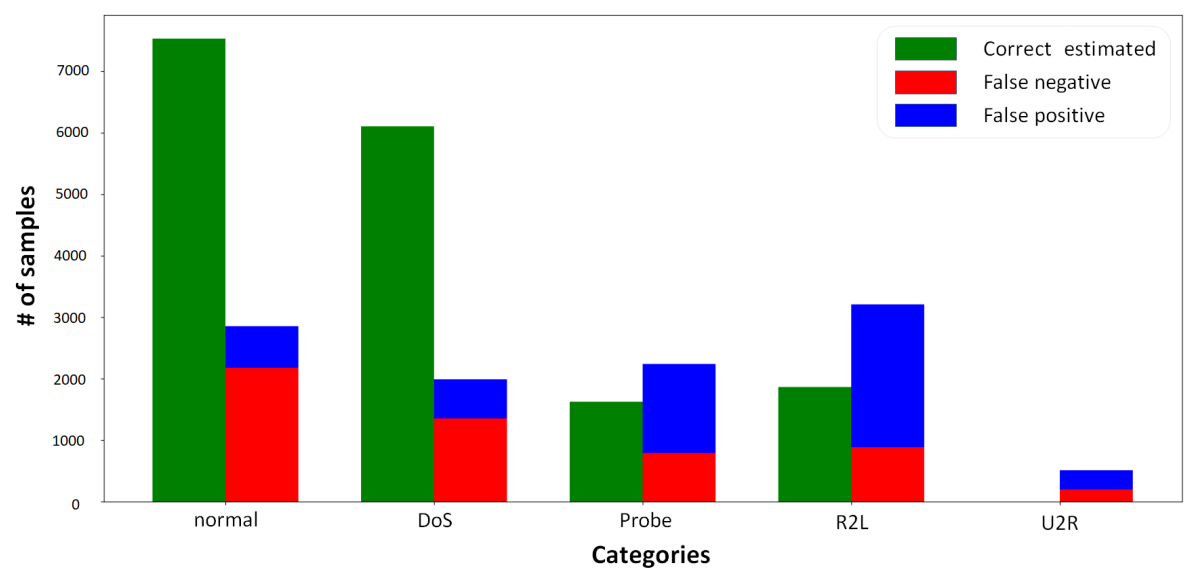

Figure 4.14: NearMiss1, F1 = 0.7602

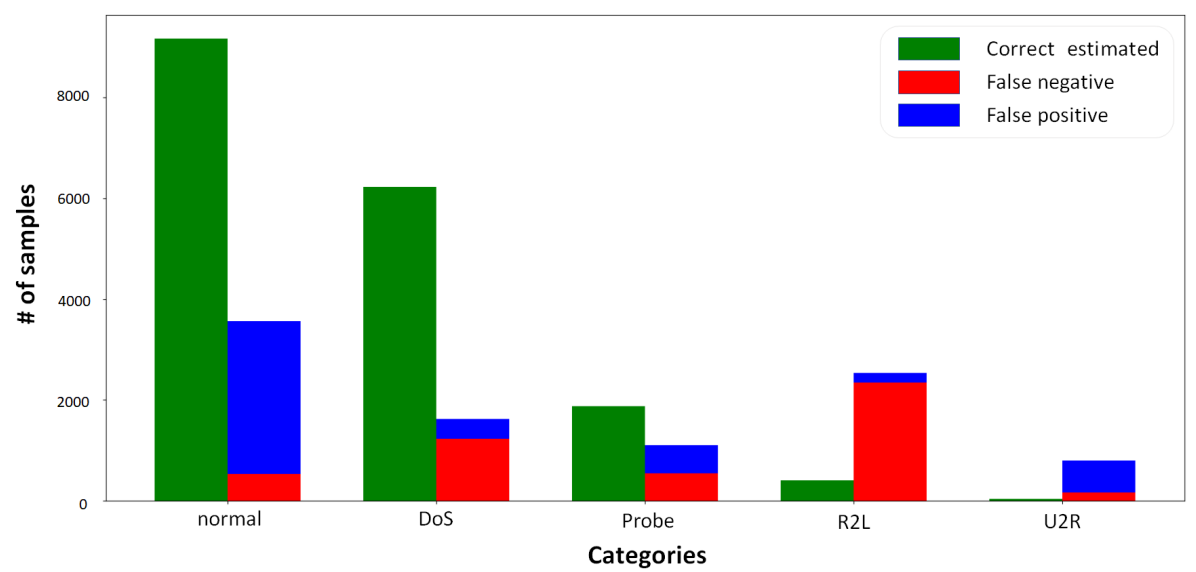

Figure 4.15: NearMiss2, F1 $=0.7856$

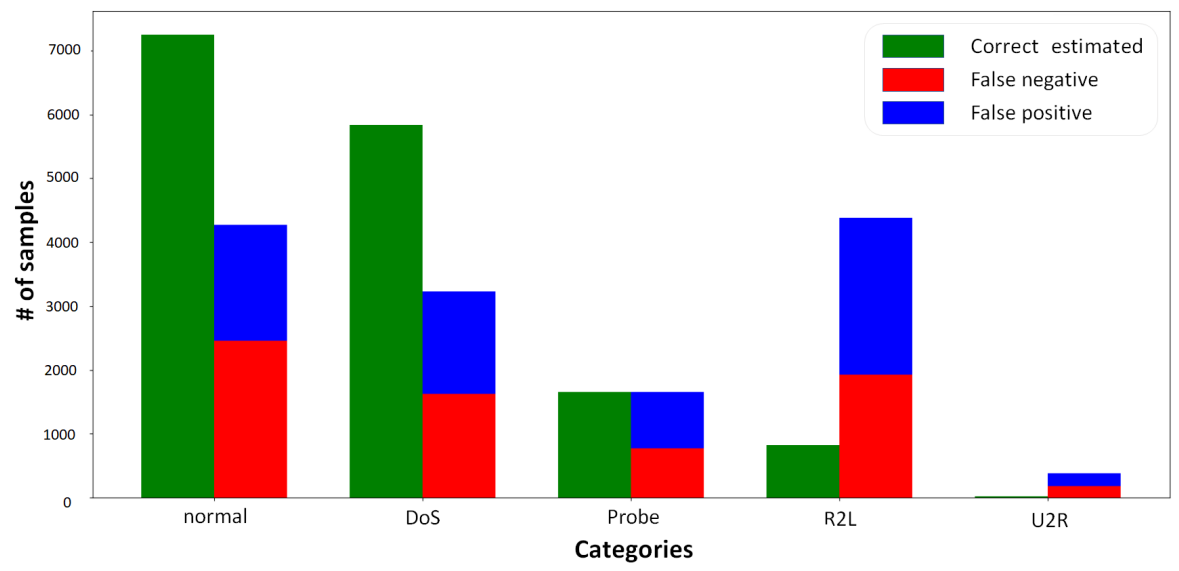

Figure 4.16: ROS, F1 $=0.6932$ 
positive rates. More specifically, NearMiss1 has lower normal prediction accuracy than NearMiss2. This is due to the fact that NearMiss1 keeps the majority samples that are closest to the nearest minority samples, which means rather keeping the whole identify of the majority class, it only keeps a cluster of samples. Simultaneously, since majority classes are in a cluster form and does not cover a wide range of area, more predictions are made for the minority classes, which results in a higher number of correct estimates. On the other hand, NearMiss2 has descent performance on the majority classes NORMAL, DoS, and PROBE, but lower number of correct estimates in R2L. This is due to the fact that NearMiss2 keeps the majority samples that are closest to the furthest minority samples. Instead of forming a cluster-like group, it forms a line-like shape. The advantage is having more accurate majority classes' predictions, but gives less predictions on the minority classes. Lastly, we can observe that ROS suffers in performance from both majority and minority classes. The fact that duplicating minority samples leads to an inferior result. 


\section{Chapter 5}

\section{Conclusion and Future Work}

IDS is a critical service that monitors networks for malicious activities such as security attacks. Difficulties have been imposed due to the network's complex and dynamic environment, which could severely affect the performance of existing IDS software. These difficulties include unbalanced, complex and asymmetric datasets. This dissertation modified the framework of AE-RL by imposing sampling techniques and specifically investigated the performance of joining SMOTE with the AE-RL framework. RL algorithms are mostly successful in other domains such as videogames, strategy games, robotics, finance, resource management, chemistry, web system configuration, etc. Yet it is very new to IDS because rewards are generally difficult to be defined.

In this dissertation, we not only adopt RL to detect anomalies including anomaly-based intrusions in networks, but also improves the class-imbalance problem which is pervasive to datasets in many domains. Our algorithm takes a labelled dataset as input, then provides a RL framework based on an adversarial strategy. Sampling techniques are implemented for better data selection in order to achieve the best possible classification results. Additionally, we propose a feature-selection mechanism during the data selection phase so that the simulated data still capture 
their main characteristics.

In summary, we present a joined framework of supervised learning, adversarial RL and sampling techniques, which results in an increasing performance of intrusion prediction. Our proposed model leverages a thorough training process on dynamic data environment. The experiment results obtained provide a thorough performance trending analysis of AESMOTE to display the optimal number of generating samples in practice and a comparative analysis of AESMOTE with AE-RL, fully displaying the benefit of our model. Finally, the comparative evaluation results demonstrate that the proposed AESMOTE has a better prediction performance than other sampling techniques such as ROS, NearMiss1 and NearMiss2.

For future work, we plan to expand the work load of environmental agent. First, we will introduce "difficulty levels" to the dataset using existing supervised learning techniques. Based on its performance, the "difficulty levels" will be further distributed into multiple partitions. By setting up multiple environmental agents, we provide a multi-adversarial strategy for data selection. The advantage of this idea is to further identifies the "weakness" of the current classifier agents, which further balances the dataset. 


\section{List of References}

[1] G. Caminero and B. Lopez-Martin, M. Carro, "Adversarial environment reinforcement learning algorithm for intrusion detection," Computer Networks, vol. 159, pp. 96-109, 2019.

[2] L. Dhanabal and S. P. Shantharajah, "A study on nsl-kdd dataset for intrusion detection system based on classification algorithms," International Journal of Advanced Research in Computer and Communication Engineering, vol. 4, no. 6, 2015.

[3] G. Fernandes, J. Rodrigues, L. Carvalho, J. Al-Muhtadi, and M. Proença, "A comprehensive survey on network anomaly detection," Telecommunication Systems, vol. 70, no. 3, pp. 447-489, 2019.

[4] M. Jaladi and N. Ghaffarzadegan, "Cybersecurity in hospitals: A systematic, organizational perspective," Journal of Medical Internet Research, vol. 20, no. 5, p. e10059, 2018.

[5] E. Perakslis, "Cybersecurity in health care," The New England Journal of Medicine, pp. 295-297, 2014.

[6] I. Brownm, "The law and economics of cybersecurity," Law Quarterly Review, vol. 123, pp. 172-175, 2007.

[7] T. Moore, "The economics of cybersecurity: Principles and policy options," International Journal of Critical Infrastructure Protection, vol. 3, pp. 103-117, 2010 .

[8] M. Warner, "Cybersecurity: A pre-history," Intelligence and National Security, vol. 27, no. 5, pp. 781-799, 2012.

[9] B. Peters, "Security considerations in a multi-programmed computer system," in Proceedings of the Spring Joint Computer Conference (AFIPS '67), (New York, USA), 1967. 
[10] W. Ware, "Report of defense science board task force on computer security," Security Controls for Computer Systems, 1970.

[11] R. Schell, "Computer security: The achilles' heel of the electronic air force?," Air $\&$ Space Power Journal, vol. 30, no. 2, 1979.

[12] K. Scarfone, "Guide to intrusion detection and prevention systems (idps)," Computer Security Resource Center, 2012.

[13] V. V. Phoha, Internet security dictionary. Springer-Verlag New York, 2002.

[14] C. C. Aggarwal, Outlier Analysis. Springer International Publishing, 2012.

[15] R. Chalapathy and S. Chawla, "Deep learning for anomaly detection: a survey." https://arxiv.org/abs/1901.03407, 2019.

[16] A. of Certified Fraud Examiners, "Report to the nations on occupational fraud and abuse," 2002.

[17] A. Abdallah, M. A. Maarof, and A. Zainal, "Fraud detection system: A survey," Journal of Network and Computer Applications, vol. 68, pp. 90-113, 2016.

[18] R. Roy and K. George, "Detecting insurance claims fraud using machine learning techniques," in 2017 International Conference on Circuit ,Power and Computing Technologies (ICCPCT), (Kollam, India), 2013.

[19] H. Joudaki, A. Rashidian, B. Minaei-Bidgoli, M. Mahmoodi, B. Gerali, M. Nasiri, and M. Arab, "Using data mining to detect health care fraud and abuse: A review of literature," Global Journal of Health Science, vol. 7, no. 1, pp. 194-202, 2015.

[20] R. Bauder and T. Khoshgoftaar, "Medicare fraud detection using machine learning methods," in 2017 16th IEEE International Conference on Machine Learning and Applications (ICMLA), (Cancun, Mexico), 2017.

[21] X. Zhou, S. Cheng, M. Zhu, C. Guo, S. Zhou, P. Xu, Z. Xue, and W. Zhang, "A state of the art survey of data mining-based fraud detection and credit scoring," in 2018 2nd International Conference on Material Engineering and Advanced Manufacturing Technology (MEAMT 2018), (Beijing, China), 2018.

[22] K. Tripathi and M. Pavaskar, "Survey on credit card fraud detection methods," International Journal of Emerging Technology and Advanced Engineering, vol. 2, no. 11, pp. 721-726, 2012. 
[23] M. Akhter and M. Ahamad, "Detecting telecommunication fraud using neural networks through data mining," International Journal of Scientific Engineering Research, vol. 3, no. 3, pp. 139-143, 2012.

[24] S. Min, B. Lee, and S. Yoon, "Deep learning in bioinformatics," Briefing in Bioinformatics, vol. 18, no. 5, pp. 851-869, 2017.

[25] M. Yousefi-Azar, V. Varadharajan, L. Hamey, and U. Tupakula, "Autoencoderbased feature learning for cyber security applications," in 2017 International Joint Conference on Neural Networks (IJCNN), (Anchorage, USA), 2017.

[26] B. Kolosnjaji, A. Demontis, B. Biggio, D. Maiorca, G. Giacinto, C. Eckert, and F. Roli, "Adversarial malware binaries: Evading deep learning for malware detection in executables," in 2018 26th European Signal Processing Conference (EUSIPCO), (Rome, Italy), 2018.

[27] I. Rosenburg, G. Sicard, and E. David, "End-to-end deep neural networks and transfer learning for automatic analysis of nation-state malware," Entropy, vol. 20 , no. 5 , p. 390, 2018.

[28] O. David and N. Netanyahu, "Deepsign: Deep learning for automatic malware signature generation and classification," in 2015 International Joint Conference on Neural Networks (IJCNN), (Killarney, Ireland), 2015.

[29] S. Tobiyama, Y. Yamaguchi, H. Hasegawa, H. Shimada, M. Akiyama, and T. Yagi, "A method for estimating process maliciousness with seq2seq model," in 2018 International Conference on Information Networking (ICOIN), (Chiang Mai, Thailand), 2018.

[30] J. Kim, S. Bu, and S. Cho, "Zero-day malware detection using transferred generative adversarial networks based on deep autoencoders," Information Sciences, vol. 460-461, pp. 83-102, 2018.

[31] H. HaddadPajouh, A. Dehghantanha, R. Khayami, and K. Choo, "A deep recurrent neural network based approach for internet of things malware threat hunting," Future Generation Computer Systems, vol. 85, pp. 88-96, 2018.

[32] G. Akpakwu, B. Silva, G. Hancke, and A. Abu-Mahfouz, "A survey on 5g networks for the internet of things: Communication technologies and challenges," IEEE Access, vol. 6, pp. 3619-3647, 2017. 
[33] D. Ramotsoela, A. Abu-Mahfouz, and G. Hancke, "A survey of anomaly detection in industrial wireless sensor networks with critical water system infrastructure as a case study," Sensors, vol. 18, no. 8, p. 2491, 2018.

[34] S. Rajasegarar, C. Leckie, and M. Palaniswami, "Anomaly detection in wireless sensor networks," IEEE Wireless Communications, vol. 15, no. 4, pp. 34-40, 2008 .

[35] M. Xie, J. Hu, S. Guo, and A. Zomaya, "Distributed segment-based anomaly detection with kullback-leibler divergence in wireless sensor networks," IEEE Transactions on Information Forensics and Security, vol. 12, no. 1, pp. 101-110, 2016 .

[36] M. Xie, J. Hu, S. Han, and H. Chen, "Scalable hypergrid k-nn-based online anomaly detection in wireless sensor networks," IEEE Transactions on Parallel and Distributed Systems, vol. 24, no. 8, pp. 1661-1670, 2012.

[37] B. Kiran, D. Thomas, and R. Parakkal, "An overview of deep learning based methods for unsupervised and semi-supervised anomaly detection in videos," Journal of Imaging, vol. 4, no. 2, p. 36, 2018.

[38] L. Dong, Y. Zhang, C. Wen, and H. Wu, "Camera anomaly detection based on morphological analysis and deep learning," in 2016 IEEE International Conference on Digital Signal Processing (DSP), (Beijing, China), 2016.

[39] W. Luo, W. Liu, and S. Gao, "A revisit of sparse coding based anomaly detection in stacked rnn framework," in 2017 IEEE International Conference on Computer Vision (ICCV), (Venice, Italy), 2017.

[40] D. Chianucci and A. Savakis, "Unsupervised change detection using spatial transformer networks," in 2016 IEEE Western New York Image and Signal Processing Workshop (WNYISPW), (Rochester, USA), 2016.

[41] M. Ravanbakhsh, E. Sangineto, M. Nabi, and N. Sebe, "Training adversarial discriminators for cross-channel abnormal event detection in crowds," in 2019 IEEE Winter Conference on Applications of Computer Vision (WACV), (Waikoloa Village, USA), 2019.

[42] M. Elrawy, A. Awad, and H. Hamed, "Intrusion detection systems for iot-based smart environments: a survey," Journal of Cloud Computing, vol. 7, no. 21, 2018. 
[43] J. King and A. Awad, "A distributed security mechanism for resourceconstrained iot devices," An International Journal of Computing and Informatics, vol. 40, no. 1, 2016.

[44] S. Faghih-Roohi, S. Hajizadeh, A. Núñez, R. Babuska, and B. Schutter, "Deep convolutional neural networks for detection of rail surface defects," in 2016 International Joint Conference on Neural Networks (IJCNN), (Vancouver, Canada), 2016.

[45] J. Inoue, Y. Yamagata, Y. Chen, C. Poskitt, and J. Sun, "Anomaly detection for a water treatment system using unsupervised machine learning," in 2017 IEEE International Conference on Data Mining Workshops (ICDMW), (New Orleans, USA), 2017.

[46] Y. Yuan and K. Jia, "A distributed anomaly detection method of operation energy consumption using smart meter data," in 2015 International Conference on Intelligent Information Hiding and Multimedia Signal Processing (IIH-MSP), (Adelaide, Australia), 2015.

[47] F. Lodhi, S. Hasan, O. Hasan, and F. Awwadl, "Power profiling of microcontroller's instruction set for runtime hardware trojans detection without golden circuit models," in Design, Automation Test in Europe Conference Exhibition (DATE), 2017, (Lausanne, Switzerland), 2017.

[48] L. Banjanovic-Mehmedovic, A. Hajdarevic, M. Kantardzic, F. Mehmedovic, and I. Dzananovic, "Neural network-based data-driven modelling of anomaly detection in thermal power plant," Automatika (Journal for Control, Measurement, Electronics, Computing and Communications), vol. 58, no. 1, pp. 69-79, 2017.

[49] D. Atha and M. Jahanshahi, "Evaluation of deep learning approaches based on convolutional neural networks for corrosion detection," Structural Health Monitoring, vol. 17, no. 5, pp. 1110-1128, 2017.

[50] F. Wang, J. Kerekes, Z. Xu, and Y. Wang, "Residential roof condition assessment system using deep learning," Journal of Applied Remote Sensing, vol. 12, no. 1, 2017.

[51] J. Valenzuela, J. Wang, and N. Bissinger, "Real-time intrusion detection in power system operations," IEEE Transactions on Power Systems, vol. 28, no. 2, pp. 1052-1062, 2019. 
[52] M. Aloqaily, S. Otoum, I. Ridhawi, and Y. Jararweh, "An intrusion detection system for connected vehicles in smart cities," Ad Hoc Networks, vol. 90, 2019.

[53] X. Chen, L. Zhang, Y. Liu, and C. Tang, "Ensemble learning methods for power system cyber-attack detection," in 2018 the 3rd IEEE International Conference on Cloud Computing and Big Data Analysis, (Changsha, China), 2018.

[54] Y. Li, R. Qiu, and S. Jing, "Intrusion detection system using online sequence extreme learning machine (os-elm) in advanced metering infrastructure of smart grid," PLoS ONE, vol. 13, no. 2, 2018.

[55] P. Zeng and P. Zhou, "Intrusion detection in scada system: A survey," ICSEE 2018, IMIOT 2018: Intelligent Computing and Internet of Things, vol. 924, pp. 342-351, 2018.

[56] C. Li and M. Qiu, Reinforcement Learning for Cyber-physical Systems with Cybersecurity Case Studies. New York, USA, 2019.

[57] X. Zhang, C. Li, and W. Zheng, "Intrusion prevention system design," in The Fourth International Conference onComputer and Information Technology (CIT'04), (Wuhan, China), 2015.

[58] E. Skoudis, "Malware: Fighting malicious code," Prentice Hall, 2003.

[59] C. Day, "Chapter 5 - intrusion prevention and detection systems," Managing Information Security (Second Edition), pp. 119-142, 2013.

[60] P. Gutman, "World's most powerful supercomputer goes online," Full Disclosure, 2007.

[61] C. Wysopal, C. Eng, and T. Shields, "Static detection of application backdoors," Datenschutz und Datensicherheit(DuD), vol. 34, pp. 149-155, 2010.

[62] R. Sutten and G. Andrew, Reinforcement Learning: An Introduction. 20142015.

[63] C. Watkins, "Q-learning," Machine Learning, vol. 8, pp. 279-292, 1992.

[64] A. Farahmand, A. Shademan, M. Jägersand, and Szepesvári, "Model-based and model-free reinforcement learning for visual serving," in 2009 IEEE International Conference on Robotics and Autom ation, (Kobe, Japan), 2009. 
[65] C. Huang, Y. Wu, Y. Zuo, K. Pei, and G. Min, "Towards experienced anomaly detector through reinforcement learning," in Proceedings of the Thirty-Second AAAI Conference on Artificial Intelligence (AAAI-18), (Hilton New Orleans Riverside, USA), 2018.

[66] J. Schmidhuber, "Deep learning in neural networks: An overview," Neural Networks, vol. 61, pp. 85-117, 2015.

[67] A. Pawar and M. Mahindraker, "A comprehensive survey on online anomaly detection," International Journal of Computer Applications, vol. 119, no. 17, pp. 41-45, 2015.

[68] A. Lazaric, M. Restelli, and A. Bonarini, "Reinforcement learning in continuous action spaces through sequential monte carlo methods," Advances in Neural Information Processing Systems, 2007.

[69] G. Tesauro, "Temporal difference learning of backgammon strategy," Machine Learning Proceedings 1992, pp. 451-457, 1992.

[70] C. Li, M. Wang, and Q. Yuan, "A multi-agent reinforcement learning using actor-critic methods," in 2008 International Conference on Machine Learning and Cybernetics, (Kunming, China), 2008.

[71] C. X. Ling and V. S. Sheng, Class Imbalance Problem. Springer, Boston, MA, 2011.

[72] N. Japkowicz, "Concept-learning in the presence of between-class and withinclass imbalances," Canadian AI 2001: Advances in Artificial Intelligence, vol. 2056, pp. 67-77, 2001.

[73] J. L. Leevy, T. M. Khoshgoftaar, B. R. A., and S. N., "A survey on addressing high-class imbalance in big data," Journal of Big Data, vol. 5, no. 42, 2018.

[74] J. Wood, "Methodology for dealing with duplicate study effects in a metaanalysis," Organizational Research Methods, vol. 11, no. 1, pp. 79-95, 2007.

[75] N. Görnitz, M. Kloft, K. Rieck, and U. Brefeld, "Toward supervised anomaly detection," Journal of Artificial Intelligence Research, vol. 46, pp. 235-262, 2013.

[76] E. Min, J. Long, Q. Liu, J. Cui, Z. Cai, and J. Ma, "Su-ids: A semi-supervised and unsupervised framework for network intrusion detection," Cloud Computing and Security, vol. 11065, pp. 322-334, 2018. 
[77] J. Andrews, E. Morton, and L. Griffin, "Detecting anomalous data using autoencoders," International Journal of Machine Learning and Computing, vol. 6, no. 1, 2016.

[78] M. Goldstein and S. Uchida, "A comparative evaluation of unsupervised anomaly detection algorithms for multivariate data," PLOS ONE, vol. 11, no. 4, pp. 1-31, 2016.

[79] G. Yuan, B. Li, Y. Yao, and S. Zhang, "A deep learning enabled subspace spectral ensemble clustering approach for web anomaly detection," (Anchorage, USA), pp. 3896-3903, 2017.

[80] J. Canady, "Next generation intrusion detection: Autonomous reinforcement learning of network attacks," in In Proceedings of the 23rd National Information Systems Secuity Conference, (Baltimore, USA), 2000.

[81] S. Shamshirband, N. B. Anuar, M. L. M. Kiah, and S. Misra, "Anomaly detection using fuzzy q-learning algorithm," Acta Polytechnica Hungarica, vol. 11, no. 8, 2014.

[82] A. Feizollah, S. Shamshirband, N. B. Anuar, R. Salleh, and M. L. MatKiah, "Anomaly detection using cooperative fuzzy logic controller," FIRA2013: Intelligent Robotics Systems: Inspiring the NEXT, vol. 376, 2013.

[83] N. Sengupta, J. Sen, J. Sil, and M. Saha, "Designing of on line intrusion detection system using rough set theory and q-learning algorithm," Neurocomputing, vol. 111, pp. 161-168, 2013.

[84] H. Zhang, X. Yu, P. Ren, C. Luo, and G. Min, "Deep adversarial learning in intrusion detection: A data augmentation enhanced framework," arXiv, 2019.

[85] X. Xue, "Sequential anomaly detection based on temporal-difference learning: Principles, models and case studies," Applied Soft Computing, vol. 10, no. 3, pp. $859-867,2010$.

[86] X. Xu and L. Y., "A kernel-based reinforcement learning approach to dynamic behavior modeling of intrusion detection," Advances in Neural Networks - ISNN200\%, vol. 4491, pp. 455-464, 2007.

[87] J. Rafati and C. D. Noelle, "Learning representations in model-free hierarchical reinforcement learning," AAAI-19/IAAI-19/EAAI-19 Proceedings, vol. 33, no. 1, 2019. 
[88] C. Zhong, M. C. Gursoy, and S. Velipasalar, "Deep actor-critic reinforcement learning for anomaly detection."

[89] M. Li, Y. Sun, H. Lu, S. Maharjan, and Z. Tian, "Deep reinforcement learning for partially observable data poisoning attack in crowdsensing systems," IEEE Internet of Things Journal, 2020.

[90] Z. Tian, C. Luo, J. Qiu, X. Du, and M. Guizani, "A distributed deep learning system for web attack detection on edge devices," IEEE Transactions on Industrial Informatics, vol. 16, no. 3, pp. 1963-1971, 2020.

[91] Z. Tian, X. Gao, S. Su, and J. Qiu, "Vcash: A novel reputation framework for identifying denial of traffic service in internet of connected vehicles," IEEE Internet of Things Journal, 2020.

[92] Z. Tian, W. Shi, Y. Wang, C. Zhu, X. Du, S. Su, Y. Sun, and N. Guizani, "Realtime lateral movement detection based on evidence reasoning network for edge computing environment," IEEE Transactions on Industrial Informatics, vol. 15, no. 7, pp. 4285-4294, 2019.

[93] Z. Tian, S. Su, W. Shi, X. Du, M. Guizani, and X. Yu, "A data-driven method for future internet route decision modeling," Future Generation Computer Systems, vol. 95, pp. 212-220, 2019.

[94] T. Raeder, G. Forman, and N. Chawala, "Learning from imbalanced data: Evaluation matters," Data Mining: Foundations and Intelligent Paradigms, vol. 23, pp. 315-331, 2012.

[95] Y. Kamei, A. Monden, S. Matsumoto, T. Kakimoto, and K. Matsumoto, "The effects of over and under sampling on fault-prone module detection," in First International Symposium on Empirical Software Engineering and Measurement (ESEM 2007), (Madrid, Spain), 2007.

[96] N. Chawla, "C4.5 and imbalanced data sets: Investigating the effect of sampling method, probabilistic estimate, and decision tree structure," in Proceedings of the Twentieth International Conference on Machine Learning, (Washington, USA), 2003.

[97] B. Yap, K. Rani, H. Rahman, S. Fong, Z. Khairudin, and N. Abdullah, "An application of oversampling, undersampling, bagging and boosting in handling imbalanced datasets," in Proceedings of the First International Conference on Advanced Data and Information Engineering (DaEng-2013), (Singapore), 2003. 
[98] S. J. Yen and Y. S. Lee, "Under-sampling approaches for improving prediction of the minority class in an imbalanced dataset," Intelligent Control and Automation, vol. 344, pp. 731-740, 2006.

[99] L. Bao, C. Juan, J. Li, and Y. Zhang, "Boosted near-miss under-sampling on svm ensembles for concept detection in large-scale imbalanced datasets," Neurocomputing, vol. 172, pp. 198-206, 2016.

[100] N. Chawla, K. Bowyer, L. Hall, and W. Kegelmeyer, "Smote: Synthetic minority over-sampling technique," Journal of Artificial Intelligence Research, vol. 16, pp. 321-357, 2002.

[101] R. Blagus and L. Lusa, "Smote for high-dimensional class-imbalanced data," Blagus and Lusa BMC Bioinformatics, vol. 14, p. 106, 2013.

[102] H. Han, W. Wang, and B. Mao, "Borderline-smote: A new over-sampling method in imbalanced data sets learning," in ICIC 2005: Advances in Intelligent Computing, (Berlin, Heidelberg), 2005.

[103] N. Chawla, A. Lazarevic, L. Hall, and K. Bowyer, "Smoteboost: Improving prediction of the minority class in boosting," in 7th European Conference on Principles and Practice of Knowledge Discovery in Databases (PKDD), (Dubrovnik, Croatia), pp. 107-119, 2003.

[104] H. He, Y. Bai, E. Garcia, and S. Li, "Adasyn: Adaptive synthetic sampling approach for imbalanced learning," in 2008 IEEE International Joint Conference on Neural Networks (IEEE World Congress on Computational Intelligence), (Hong Kong, China), 2008.

[105] M. Tavallaee, E. Bagheri, W. Lu, and A. Ghorbani, "A detailed analysis of the kdd cup 99 data set," in Proceedings of the 2009 IEEE Symposium on Computational Intelligence in Security and Defense Applications (CISDA2009), (Ottawa, Canada), 2009.

[106] S. Mousavi, M. Schukat, and E. Howley, "Deep reinforcement learning: An overview," in IntelliSys 2016: Proceedings of SAI Intelligent Systems Conference (IntelliSys), vol. 16, (Springer, Cham), pp. 426-440, 2017.

[107] H. Hasselt, A. Guez, and D. Silver, "Deep reinforcement learning with double q-learning," in Thirtieth AAAI Conference on Artificial Intelligence, (Phoenix, USA), 2016. 
[108] T. Lai, "Stochastic approximation," The Annals of Statistics, vol. 31, no. 2, pp. 391-406, 2003.

[109] D. Powers, "Evaluation: from precision, recall and f-measure to roc, informedness, markedness and correlation," Journal of Machine Learning Technologies, vol. 2, no. 1, pp. 37-63, 2011.

[110] M. Abadi, P. Barham, J. Chen, Z. Chen, A. Davis, J. Dean, M. Devin, S. Ghemawat, G. Irving, M. Isard, M. Kudlur, J. Levenberg, R. Monga, S. Moore, D. G. Murray, B. Steiner, P. Tucker, V. Vasudevan, P. Warden, M. Wicke, Y. Yu, and X. Zheng, "Tensorflow: A system for large-scale machine learning," in 12th USENIX Symposium on Operating Systems Design and Implementation (OSDI 16), (Savannah, GA), pp. 265-283, 2016.

[111] A. Gulli and S. Pal, Deep Learning with Keras. Packt Publishing, 2017.

[112] W. McKinney, Python for Data Analysis. O'Reilly Media, Inc., 2012.

[113] F. Pedregosa, G. Varoquaux, A. Gramfort, V. Michel, B. Thirion, O. Grisel, M. Blondel, P. Prettenhofer, R. Weiss, V. Dubourg, J. Vanderplas, A. Passos, and D. Cournapeau, "Scikit-learn: Machine learning in python," Journal of Machine Learning Research, vol. 12, pp. 2825-2830, 2011.

[114] M. Bhuyan, D. Bhattacharyya, and J. Kalita, "Network anomaly detection: Methods, systems and tools," IEEE Communications Surveys 83 Tutorials, vol. 16, no. 1, pp. 303-306, 2014. 